\title{
FORWARD-BACKWARD-HALF FORWARD ALGORITHM FOR SOLVING MONOTONE INCLUSIONS
}

\author{
LUIS M. BRICEÑO-ARIAS* AND DAMEK DAVIS ${ }^{\dagger}$
}

\begin{abstract}
Tseng's algorithm finds a zero of the sum of a maximally monotone operator and a monotone continuous operator by evaluating the latter twice per iteration. In this paper, we modify Tseng's algorithm for finding a zero of the sum of three operators, where we add a cocoercive operator to the inclusion. Since the sum of a cocoercive and a monotone-Lipschitz operator is monotone and Lipschitz, we could use Tseng's method for solving this problem, but implementing both operators twice per iteration and without taking into advantage the cocoercivity property of one operator. Instead, in our approach, although the continuous monotone operator must still be evaluated twice, we exploit the cocoercivity of one operator by evaluating it only once per iteration. Moreover, when the cocoercive or continuous-monotone operators are zero it reduces to Tseng's or forward-backward splittings, respectively, unifying in this way both algorithms. In addition, we provide a preconditioned version of the proposed method including non self-adjoint linear operators in the computation of resolvents and the single-valued operators involved. This approach allows us to also extend previous variable metric versions of Tseng's and forward-backward methods and simplify their conditions on the underlying metrics. We also exploit the case when non self-adjoint linear operators are triangular by blocks in the primal-dual product space for solving primal-dual composite monotone inclusions, obtaining Gauss-Seidel type algorithms which generalize several primal-dual methods available in the literature. Finally we explore applications to the obstacle problem, Empirical Risk Minimization, distributed optimization and nonlinear programming and we illustrate the performance of the method via some numerical simulations.
\end{abstract}

Key words. Convex optimization, forward-backward splitting, monotone operator theory, sequential algorithms, Tseng's splitting.

AMS subject classifications. 47H05, 65K05, 65K15, 90C25

1. Introduction. This paper is devoted to the numerical resolution of following problem.

Problem 1. Let $X$ be a nonempty closed convex subset of a real Hilbert space $\mathcal{H}$, let $A: \mathcal{H} \rightarrow 2^{\mathcal{H}}$ and $B_{2}: \mathcal{H} \rightarrow 2^{\mathcal{H}}$ be maximally monotone operators, with $B_{2}$ single valued in $\operatorname{dom} B_{2} \supset \operatorname{dom} A \cup X$, and let $B_{1}: \mathcal{H} \rightarrow \mathcal{H}$ be $\beta$-cocoercive ${ }^{1}$, for some $\beta>0$. Moreover assume that $B_{2}$ is continuous on $\operatorname{dom} A \cup X$ and that $A+B_{2}$ is maximally monotone. The problem is to

$$
\text { find } \quad x \in X \text { such that } 0 \in A x+B_{1} x+B_{2} x \text {, }
$$

under the assumption that the set of solutions to (1.1) is nonempty.

The wide variety of applications of Problem 1 involving optimization problems, variational inequalities, partial differential equations, image processing, saddle point problems, game theory, among others can be explored in [3, 20] and the references therein. As an important application, consider the case of composite optimization problems of the form

$$
\underset{\mathrm{x} \in \mathrm{H}}{\operatorname{minimize}} \mathrm{f}(\mathrm{x})+\mathrm{g}(\mathrm{Lx})+\mathrm{h}(\mathrm{x})
$$

* Department of Mathematics, Universidad Técnica Federico Santa María, Santiago, Chile, (luis.briceno@usm.cl)

$\dagger$ School of Operations Research and Information Engineering, Cornell University, Ithaca, NY 14850 (dsd95@cornell.edu)

${ }^{1}$ An operator $C: \mathcal{H} \rightarrow \mathcal{H}$ is $\beta$-cocoercive for some $\beta>0$ provided that $\langle C x-C y, x-y\rangle \geq$ $\beta\|C x-C y\|^{2}$. 
where $\mathrm{H}$ and $\mathrm{G}$ are real Hilbert spaces, $\mathrm{L}: \mathrm{H} \rightarrow \mathrm{G}$ is linear and bounded, $\mathrm{f}: \mathrm{H} \rightarrow$ $(-\infty, \infty]$ and $\mathrm{g}: \mathrm{G} \rightarrow(-\infty, \infty]$ are lower semicontinuous, convex, and proper, and $\mathrm{h}: \mathrm{H} \rightarrow \mathbb{R}$ is convex differentiable with $\beta^{-1}$-Lipschitz gradient. Since $g$ may be non smooth, primal algorithms in this context need to evaluate $\operatorname{prox}_{\mathrm{g} \circ L}$ or invert $L$ which can be costly numerically. In order to overcome this difficulty, fully split primal-dual algorithms are proposed, e.g., in $[8,21,42]$, in which only $\operatorname{prox}_{\mathrm{g}}, L$, and $L^{*}$ are computed. These algorithms follow from the first order optimality conditions of (1.2), which, under qualification conditions, can be written as Problem 1 with

$$
X=\mathcal{H}=\mathrm{H} \times \mathrm{G}, \quad A=\partial \mathrm{f} \times \partial \mathrm{g}^{*}, \quad B_{1}=\nabla \mathrm{h} \times\{0\}, \quad B_{2}=\left[\begin{array}{cc}
0 & \mathrm{~L}^{*} \\
-\mathrm{L} & 0
\end{array}\right],
$$

where we point out that $B_{2}$ is monotone and Lipschitz but not cocoercive, because it is skew linear and, for every $x \in \mathcal{H},\left\langle x \mid B_{2} x\right\rangle=0$. We have that, for any solution $x=$ $\left(\mathrm{x}_{1}^{*}, \mathrm{x}_{2}^{*}\right) \in \operatorname{zer}\left(A+B_{1}+B_{2}\right), \mathrm{x}_{1}^{*}$ solves $(1.2)$, where we denote zer $T=\{x \in \mathcal{H} \mid 0 \in T x\}$ for any set valued operator $T: \mathcal{H} \rightarrow 2^{\mathcal{H}}$. A method proposed in [42] solves (1.2) in a more general context by using forward-backward splitting $(\mathrm{FB})$ in the product space with the metric $\langle\cdot \mid \cdot\rangle_{V}=\langle V \cdot \mid \cdot\rangle$ for the operators $V^{-1}\left(A+B_{2}\right)$ and $V^{-1} B_{1}$ with a specific choice of self-adjoint strongly monotone linear operator $V$. We recall that the forward-backward splitting $[16,10,32,25]$ finds a zero of the sum of a maximally monotone and a cocoercive operator, which is a particular case of Problem 1 when $X=\mathcal{H}$ and $B_{2}=0$. This method provides a sequence obtained from the fixed point iteration of the nonexpansive operator (for some $\gamma \in] 0,2 \beta[$ )

$$
T_{\mathrm{FB}}:=J_{\gamma A} \circ\left(\mathrm{Id}-\gamma B_{1}\right),
$$

which converges weakly to a zero of $A+B_{1}$. Here Id stands for the identity map in $\mathcal{H}$ and, for every set valued operator $M: \mathcal{H} \rightarrow 2^{\mathcal{H}}, J_{M}=(\operatorname{Id}+M)^{-1}: \mathcal{H} \rightarrow 2^{\mathcal{H}}$ is the resolvent of $M$, which is single valued and nonexpansive when $M$ is maximally monotone. In the context of (1.3), the operators $V^{-1}\left(A+B_{2}\right)$ and $V^{-1} B_{1}$ are maximally monotone and $\beta$-cocoercive in the metric $\langle\cdot \mid \cdot\rangle_{V}=\langle V \cdot \mid \cdot\rangle$, respectively, which ensures the convergence of the forward-backward splitting. The choice of $V$ permits the explicit computation of $J_{V^{-1}\left(A+B_{2}\right)}$, which leads to a sequential method that generalizes the algorithm proposed in [13]. A variant for solving (1.2) in the case when $h=0$ is proposed in [27]. However, previous methods need the skew linear structure of $B_{2}$ in order to obtain an implementable method.

An example in which a non-linear continuous operator $B_{2}$ arises naturally is the convex constrained optimization problem

$$
\min _{\substack{x \in C \\ g(x) \leq 0}} f(x),
$$

where $f: \mathcal{H} \rightarrow \mathbb{R}$ is convex differentiable with $\beta^{-1}$-Lipschitz-gradient, $C \subset \mathcal{H}$ is nonempty, closed and convex, and $g: \mathcal{H} \rightarrow \mathbb{R}$ is a $\mathcal{C}^{1}$ and convex function. The Lagrangian function in this case takes the form

$$
L(x, \lambda)=\iota_{C}(x)+f(x)+\lambda g(x)-\iota_{\mathbb{R}_{+}}(\lambda),
$$

which, under standard qualification conditions can be found by solving the monotone inclusion (see [34])

$$
0 \in A(x, \lambda)+B_{1}(x, \lambda)+B_{2}(x, \lambda),
$$


where $A:(x, \lambda) \mapsto N_{C} x \times N_{\mathbb{R}_{+}} \lambda$ is maximally monotone, $B_{1}:(x, \lambda) \mapsto(\nabla f(x), 0)$ is cocoercive, and $B_{2}:(x, \lambda) \mapsto(\lambda \nabla g(x),-g(x))$ is monotone and continuous [34]. Of course, the problem can be easily extended to consider finitely many inequality and equality constraints and allow for more general lower semicontinuous convex functions than $\iota_{C}$, but we prefer the simplified version for the ease of presentation. Note that the non-linearity of $B_{2}$ does not allow to use previous methods in this context.

In the case when $B_{2}$ is $L$-Lipschitz for some $L>0$, since $B:=B_{1}+B_{2}$ is monotone and $\left(\beta^{-1}+L\right)$-Lipschitz continuous, the forward-backward-forward splitting (FBF) proposed by Tseng in [40] solves Problem 1 . This method generates a sequence from the fixed point iteration of the operator

$$
T_{\mathrm{FBF}}:=P_{X} \circ\left[(\mathrm{Id}-\gamma B) \circ J_{\gamma A} \circ(\mathrm{Id}-\gamma B)+\gamma B\right],
$$

which converges weakly to a zero of $A+B$, provided that $\gamma \in] 0,\left(\beta^{-1}+L\right)^{-1}[$. However, this approach has two drawbacks:

1. FBF needs to evaluate $B=B_{1}+B_{2}$ twice per iteration, without taking into advantage the cocoercivity property of $B_{1}$. In the particular case when $B_{2}=0$, this method computes $B_{1}$ twice at each iteration, while the forwardbackward splitting needs only one computation of $B_{1}$ for finding a zero of $A+B_{1}$. Even if we cannot ensure that FB is more efficient than FBF in this context, the cost of each iteration of FB is lower than that of FBF, especially when the computation cost of $B_{1}$ is high. This is usually the case, for instance, when $A, B_{1}$, and $B_{2}$ are as in (1.3) and we aim at solving (1.2) representing a variational formulation of some partial differential equation (PDE). In this case, the computation of $\nabla \mathrm{h}$ frequently amounts to solving a PDE, which is computationally costly.

2. The step size $\gamma$ in FBF is bounded above by $\left(\beta^{-1}+L\right)^{-1}$, which in the case when the influence of $B_{2}$ in the problem is low $\left(B_{2} \approx 0\right)$ leads to a method whose step size cannot go too far beyond $\beta$. In the case $B_{2}=0$, the step size $\gamma$ in FB is bounded by $2 \beta$. This can affect the performance of the method, since very small stepsizes can lead to slow algorithms.

In the general case when $B_{2}$ is monotone and continuous, we can also apply a version of the method in [40] which uses line search for choosing the step-size at each iteration. However, this approach share the disadvantage of computing twice $B_{1}$ by iteration and, moreover, in the line search $B_{1}$ has to be computed several times up to find a sufficiently small step-size, which can be computationally costly.

In this paper we propose a splitting algorithm for solving Problem 1 which overcomes previous drawbacks. The method is derived from the fixed point iteration of the operator $T_{\gamma}: \mathcal{H} \rightarrow \mathcal{H}$, defined by

$$
T_{\gamma}:=P_{X} \circ\left[\left(\operatorname{Id}-\gamma B_{2}\right) \circ J_{\gamma A} \circ\left(\operatorname{Id}-\gamma\left(B_{1}+B_{2}\right)\right)+\gamma B_{2}\right],
$$

for some $\gamma \in] 0, \chi(\beta, L)\left[\right.$, where $\chi(\beta, L) \leq \min \left\{2 \beta, L^{-1}\right\}$ in the case when $B_{2}$ is $L$ Lipschitz. The algorithm thus obtained implements $B_{1}$ only once by iteration and it reduces to $\mathrm{FB}$ or $\mathrm{FBF}$ when $X=\mathcal{H}$ and $B_{2}=0$, or $B_{1}=0$, respectively, and in these cases we have $\chi(\beta, 0)=2 \beta$ and $\lim _{\beta \rightarrow+\infty} \chi(\beta, L)=L^{-1}$. Moreover, in the case when $B_{2}$ is merely continuous, the step-size is found by a line search in which $B_{1}$ is only computed once at each backtracking step. These results can be found in Theorem 2.3 in Section 2. Moreover, a generalization of FB for finding a point in $X \cap \operatorname{zer}\left(A+B_{1}\right)$ can be derived when $B_{2}=0$. This can be useful when the solution is known to belong to a closed convex set $X$, which is the case, for example, in convex constrained 
minimization. The additional projection onto $X$ can improve the performance of the method (see, e.g., [9]).

Another contribution of this paper is to include in our method non self-adjoint linear operators in the computation of resolvents and other operators involved. More precisely, in Theorem 3.2 in Section 3, for an invertible linear operator $P$ (not necesarily self-adjoint) we justify the computation of $P^{-1}\left(B_{1}+B_{2}\right)$ and $J_{P^{-1} A}$, respectively. In the case when $P$ is self-adjoint and strongly monotone, the properties that $A, B_{1}$ and $B_{2}$ have with the standard metric are preserved by $P^{-1} A, P^{-1} B_{1}$, and $P^{-1} B_{2}$ in the metric $\langle\cdot \mid \cdot\rangle_{P}=\langle P \cdot \mid \cdot\rangle$. In this context, variable metric versions of $\mathrm{FB}$ and $\mathrm{FBF}$ have been developed in $[19,41]$. Of course, a similar generalization can be done for our algorithm, but we go beyond this self-adjoint case and we implement $P^{-1}\left(B_{1}+B_{2}\right)$ and $J_{P^{-1} A}$, where the linear operator $P$ is strongly monotone but non necesarily selfadjoint. The key for this implementation is the decomposition $P=S+U$, where $U$ is self-adjoint and strongly monotone and $S$ is skew linear. Our implementation follows after coupling $S$ with the monotone and Lipschitz component $B_{2}$ and using some resolvent identities valid for the metric $\langle\cdot \mid \cdot\rangle_{U}$. One of the important implications of this issue is the justification of the convergence of some Gauss-Seidel type methods in product spaces, which are deduced from our setting for block triangular linear operators $P$.

Additionally, we provide a modification of the previous method in Theorem 4.2, in which linear operators $P$ may vary among iterations. In the case when, for every iteration $k \in \mathbb{N}, P_{k}$ is self-adjoint, this feature has also been implemented for FB and FBF in $[19,41]$ but with a strong dependence between $P_{k+1}$ and $P_{k}$ coming from the variable metric approach. Instead, in the general case, we modify our method for allowing variable metrics and ensuring convergence under weaker conditions. For instance, in the case when $B_{2}=0$ and $P_{k}$ is self-adjoint and $\rho_{k}$-strongly monotone for some $\rho_{k}>0$, our condition on our FB variable metric version reduces to $(2 \beta-\varepsilon) \rho_{k}>1$ for every $k \in \mathbb{N}$. In the case when $P_{k}=\mathrm{Id} / \gamma_{k}$ this condition reduces to $\gamma_{k}<2 \beta-\varepsilon$ which is a standard assumption for $\mathrm{FB}$ with variable stepsizes. Hence, our condition on operators $\left(P_{k}\right)_{k \in \mathbb{N}}$ can be interpreted as "step-size" bounds.

Moreover, in Section 5 we use our methods in composite primal-dual inclusions, obtaining generalizations and new versions of several primal-dual methods $[13,41$, $30,17]$. We provide comparisons among methods and new bounds on stepsizes which improve several bounds in the literature. Finally, for illustrating the flexibility of the proposed methods, in Section 6 we apply them to the obstacle problem in PDE's, to empirical risk minimization, to distributed operator splitting schemes and to nonlinear constrained optimization. In the first example, we take advantage to dropping the extra forward step on $B_{1}$, which amounts to reduce the computation of a PDE by iteration. In the second example, we use non self-adjoint linear operators in order to obtain a Gauss-Seidel structure which can be preferable to parallel architectures for high dimensions. The third example illustrates how the variable metrics allowed by our proposed algorithm can be used to develop distributed operator splitting schemes with time-varying communication networks. The last example illustrates our backtracking line search procedure for nonlinear constrained optimization wherein the underlying operator $B_{2}$ is nonlinear and non Lipschitz. Finally, some numerical examples show the performance of the proposed algorithms.

2. Convergence theory. This section is devoted to study the conditions ensuring the convergence of the iterates generated recursively by $z^{k+1}=T_{\gamma_{k}} z^{k}$ for any starting point $z^{0} \in \mathcal{H}$, where, for every $\gamma>0, T_{\gamma}$ is defined in (1.7). We 
first prove that $T_{\gamma}$ is quasi-nonexpansive for a suitable choice of $\gamma$ and satisfies $\operatorname{Fix}\left(T_{\gamma}\right)=\operatorname{zer}\left(A+B_{1}+B_{2}\right) \cap X$. Using these results we prove the weak convergence of iterates $\left\{z^{k}\right\}_{k \in \mathbb{N}}$ to a solution to Problem 1.

Proposition 2.1 (Properties of $T_{\gamma}$ ). Let $\gamma>0$, assume that hypotheses of Problem 1 hold, and set $S_{\gamma}:=\left(\operatorname{Id}-\gamma B_{2}\right) \circ J_{\gamma A} \circ\left(\operatorname{Id}-\gamma\left(B_{1}+B_{2}\right)\right)+\gamma B_{2}$. Then,

1. We have $\operatorname{zer}\left(A+B_{1}+B_{2}\right) \subset$ Fix $S_{\gamma}$ and $\operatorname{zer}\left(A+B_{1}+B_{2}\right) \cap X \subset \operatorname{Fix} T_{\gamma}$. Moreover, if $B_{2}$ is L-Lipschitz in $\operatorname{dom} B_{2}$ for some $L>0$ and $\gamma<L^{-1}$ we have $\operatorname{Fix}\left(S_{\gamma}\right)=\operatorname{zer}\left(A+B_{1}+B_{2}\right)$ and $\operatorname{Fix}\left(T_{\gamma}\right)=\operatorname{zer}\left(A+B_{1}+B_{2}\right) \cap X$.

2. For all $z^{*} \in \operatorname{Fix}\left(T_{\gamma}\right)$ and $z \in \operatorname{dom} B_{2}$, by denoting $x:=J_{\gamma A}\left(z-\gamma\left(B_{1}+B_{2}\right) z\right)$ we have, for every $\varepsilon>0$,

$$
\begin{aligned}
\left\|T_{\gamma} z-z^{*}\right\|^{2} \leq & \left\|z-z^{*}\right\|^{2}-(1-\varepsilon)\|z-x\|^{2}+\gamma^{2}\left\|B_{2} z-B_{2} x\right\|^{2} \\
& -\frac{\gamma}{\varepsilon}(2 \beta \varepsilon-\gamma)\left\|B_{1} z-B_{1} z^{*}\right\|^{2}-\varepsilon\left\|z-x-\frac{\gamma}{\varepsilon}\left(B_{1} z-B_{1} z^{*}\right)\right\|^{2} .
\end{aligned}
$$

3. Suppose that $B_{2}$ is L-Lipschitz in $\operatorname{dom} A \cup X$ for some $L>0$. For all $z^{*} \in$ $\operatorname{Fix}\left(T_{\gamma}\right)$ and $z \in \operatorname{dom} B_{2}$, by denoting $x:=J_{\gamma A}\left(z-\gamma\left(B_{1}+B_{2}\right) z\right)$ we have

$$
\begin{aligned}
\left\|T_{\gamma} z-z^{*}\right\|^{2} & \leq\left\|z-z^{*}\right\|^{2}-L^{2}\left(\chi^{2}-\gamma^{2}\right)\|z-x\|^{2}-\frac{2 \beta \gamma}{\chi}(\chi-\gamma)\left\|B_{1} z-B_{1} z^{*}\right\|^{2} \\
& -\frac{\chi}{2 \beta}\left\|z-x-\frac{2 \beta \gamma}{\chi}\left(B_{1} z-B_{1} z^{*}\right)\right\|^{2}
\end{aligned}
$$

where

$$
\chi:=\frac{4 \beta}{1+\sqrt{1+16 \beta^{2} L^{2}}} \leq \min \left\{2 \beta, L^{-1}\right\} .
$$

Proof. Part 1: Let $z^{*} \in \mathcal{H}$. We have

$$
\begin{aligned}
z^{*} \in \operatorname{zer}\left(A+B_{1}+B_{2}\right) & \Leftrightarrow 0 \in A z^{*}+B_{1} z^{*}+B_{2} z^{*} \\
& \Leftrightarrow-\gamma\left(B_{1} z^{*}+B_{2} z^{*}\right) \in \gamma A z^{*} \\
& \Leftrightarrow z^{*}=J_{\gamma A}\left(z^{*}-\gamma\left(B_{1} z^{*}+B_{2} z^{*}\right)\right) .
\end{aligned}
$$

Then, since $B_{2}$ is single-valued in $\operatorname{dom} A$, if $z^{*} \in \operatorname{zer}\left(A+B_{1}+B_{2}\right)$ we have $B_{2} z^{*}=$ $B_{2} J_{\gamma A}\left(z^{*}-\gamma\left(B_{1} z^{*}+B_{2} z^{*}\right)\right)$ and, hence, $S_{\gamma} z^{*}=z^{*}$ which yields $\operatorname{zer}\left(A+B_{1}+B_{2}\right) \subset$ Fix $S_{\gamma}$. Hence, if $z^{*} \in \operatorname{zer}\left(A+B_{1}+B_{2}\right) \cap X$ then $z^{*} \in$ Fix $P_{X}$ and $z^{*} \in$ Fix $S_{\gamma}$, which yields $z^{*} \in \operatorname{Fix} P_{X} \circ S_{\gamma}=\operatorname{Fix} T_{\gamma}$. Conversely, if $B_{2}$ is $L$-Lipschitz in $\operatorname{dom} B_{2}$ and $z^{*} \in \operatorname{Fix} S_{\gamma}$ we have

$$
z^{*}-J_{\gamma A}\left(z^{*}-\gamma\left(B_{1}+B_{2}\right) z^{*}\right)=\gamma\left(B_{2} z^{*}-B_{2} J_{\gamma A}\left(z^{*}-\gamma\left(B_{1}+B_{2}\right) z^{*}\right)\right),
$$

which, from the Lipschitz continuity of $B_{2}$ yields

$$
\begin{aligned}
\left\|z^{*}-J_{\gamma A}\left(z^{*}-\gamma\left(B_{1}+B_{2}\right) z^{*}\right)\right\| & =\gamma\left\|B_{2} z^{*}-B_{2} J_{\gamma A}\left(z^{*}-\gamma\left(B_{1}+B_{2}\right) z^{*}\right)\right\| \\
& \leq \gamma L\left\|z^{*}-J_{\gamma A}\left(z^{*}-\gamma\left(B_{1}+B_{2}\right) z^{*}\right)\right\| .
\end{aligned}
$$

Therefore, if $\gamma<L^{-1}$ we deduce $z^{*}=J_{\gamma A}\left(z^{*}-\gamma\left(B_{1}+B_{2}\right) z^{*}\right)$ and from (2.4), we deduce $\operatorname{zer}\left(A+B_{1}+B_{2}\right)=$ Fix $S_{\gamma}$. Since $T_{\gamma}=P_{X} S_{\gamma}$ and $P_{X}$ is strictly quasinonexpansive, the result follows from [3, Proposition 4.49]. 
Part 2: Let $z^{*} \in \operatorname{Fix} T_{\gamma}, z \in \operatorname{dom} B_{2}$ and define $B:=B_{1}+B_{2}, y:=z-\gamma B z, x:=$ $J_{\gamma A} y$, and $z^{+}=T_{\gamma} z$. Note that $(x, y-x) \in \operatorname{gra}(\gamma A)$ and, from Part $1,\left(z^{*},-\gamma B z^{*}\right) \in$ $\operatorname{gra}(\gamma A)$. Hence, by the monotonicity of $A$ and $B_{2}$, we have $\left\langle x-z^{*}, x-y-\gamma B z^{*}\right\rangle \leq 0$ and $\left\langle x-z^{*}, \gamma B_{2} z^{*}-\gamma B_{2} x\right\rangle \leq 0$. Thus,

$$
\begin{aligned}
\left\langle x-z^{*}, x-y-\gamma B_{2} x\right\rangle & =\left\langle x-z^{*}, \gamma B_{1} z^{*}\right\rangle \\
& +\left\langle x-z^{*}, x-y-\gamma B z^{*}\right\rangle \\
& +\left\langle x-z^{*}, \gamma B_{2} z^{*}-\gamma B_{2} x\right\rangle \\
\leq & \left\langle x-z^{*}, \gamma B_{1} z^{*}\right\rangle
\end{aligned}
$$

Therefore, we have

$$
\begin{aligned}
2 \gamma\left\langle x-z^{*}, B_{2} z-B_{2} x\right\rangle & =2\left\langle x-z^{*}, \gamma B_{2} z+y-x\right\rangle+2\left\langle x-z^{*}, x-y-\gamma B_{2} x\right\rangle \\
& \leq 2\left\langle x-z^{*}, \gamma B z+y-x\right\rangle+2\left\langle x-z^{*}, \gamma B_{1} z^{*}-\gamma B_{1} z\right\rangle \\
& =2\left\langle x-z^{*}, z-x\right\rangle+2\left\langle x-z^{*}, \gamma B_{1} z^{*}-\gamma B_{1} z\right\rangle \\
& =\left\|z-z^{*}\right\|^{2}-\left\|x-z^{*}\right\|^{2}-\|z-x\|^{2}+2\left\langle x-z^{*}, \gamma B_{1} z^{*}-\gamma B_{1} z\right\rangle .
\end{aligned}
$$

In addition, by cocoercivity of $B_{1}$, for all $\varepsilon>0$, we have

$$
\begin{aligned}
2\left\langle x-z^{*}, \gamma B_{1} z^{*}-\gamma B_{1} z\right\rangle & =2\left\langle z-z^{*}, \gamma B_{1} z^{*}-\gamma B_{1} z\right\rangle+2\left\langle x-z, \gamma B_{1} z^{*}-\gamma B_{1} z\right\rangle \\
& \leq-2 \gamma \beta\left\|B_{1} z-B_{1} z^{*}\right\|^{2}+2\left\langle x-z, \gamma B_{1} z^{*}-\gamma B_{1} z\right\rangle \\
& =-2 \gamma \beta\left\|B_{1} z-B_{1} z^{*}\right\|^{2}+\varepsilon\|z-x\|^{2}+\frac{\gamma^{2}}{\varepsilon}\left\|B_{1} z-B_{1} z^{*}\right\|^{2} \\
& -\varepsilon\left\|z-x-\frac{\gamma}{\varepsilon}\left(B_{1} z-B_{1} z^{*}\right)\right\|^{2} \\
& =\varepsilon\|z-x\|^{2}-\gamma\left(2 \beta-\frac{\gamma}{\varepsilon}\right)\left\|B_{1} z-B_{1} z^{*}\right\|^{2} \\
& -\varepsilon\left\|z-x-\frac{\gamma}{\varepsilon}\left(B_{1} z-B_{1} z^{*}\right)\right\|^{2} .
\end{aligned}
$$

Hence, combining (2.5) and (2.6), it follows from $z^{*} \in X$, the nonexpansivity of $P_{X}$, and the Lipschitz property of $B_{2}$ in $\operatorname{dom} B_{2} \supset X \cup \operatorname{dom} A$ that

$$
\begin{aligned}
\left\|z^{+}-z^{*}\right\|^{2} \leq & \left\|x-z^{*}+\gamma B_{2} z-\gamma B_{2} x\right\|^{2} \\
= & \left\|x-z^{*}\right\|^{2}+2 \gamma\left\langle x-z^{*}, B_{2} z-B_{2} x\right\rangle+\gamma^{2}\left\|B_{2} z-B_{2} x\right\|^{2} \\
\leq & \left\|z-z^{*}\right\|^{2}-\|z-x\|^{2}+\gamma^{2}\left\|B_{2} z-B_{2} x\right\|^{2} \\
& +\varepsilon\|z-x\|^{2}-\gamma\left(2 \beta-\frac{\gamma}{\varepsilon}\right)\left\|B_{1} z-B_{1} z^{*}\right\|^{2}-\varepsilon\left\|z-x-\frac{\gamma}{\varepsilon}\left(B_{1} z-B_{1} z^{*}\right)\right\|^{2},
\end{aligned}
$$

and the result follows.

Part 3: It follows from (2.7) and the Lipschitz property on $B_{2}$ that

$$
\begin{aligned}
& \left\|z^{+}-z^{*}\right\|^{2} \leq\left\|z-z^{*}\right\|^{2}-L^{2}\left(\frac{1-\varepsilon}{L^{2}}-\gamma^{2}\right)\|z-x\|^{2}-\frac{\gamma}{\varepsilon}(2 \beta \varepsilon-\gamma)\left\|B_{1} z-B_{1} z^{*}\right\|^{2} \\
& -\varepsilon\left\|z-x-\frac{\gamma}{\varepsilon}\left(B_{1} z-B_{1} z^{*}\right)\right\|^{2} .
\end{aligned}
$$


In order to obtain the largest interval for $\gamma$ ensuring that the second and third terms on the right of the above equation are negative, we choose the value $\varepsilon$ so that $\sqrt{1-\varepsilon} / L=2 \beta \varepsilon$, which yields $\varepsilon=\left(-1+\sqrt{1+16 \beta^{2} L^{2}}\right)\left(8 \beta^{2} L^{2}\right)^{-1}$. For this choice of $\varepsilon$ we obtain $\chi=\sqrt{1-\varepsilon} / L=2 \beta \varepsilon$.

In the case when $B_{2}$ is merely continuous, we need the following result, which gives additional information to [40, Lemma 3.3] and allows us to guarantee the convergence of the algorithm under weaker assumptions than [40, Theorem 3.4].

Lemma 2.2. In the context of Problem 1, define, for every $z \in \operatorname{dom} B_{2}$ and $\gamma>0$,

$$
x_{z}: \gamma \mapsto J_{\gamma A}\left(z-\gamma\left(B_{1}+B_{2}\right) z\right) \quad \text { and } \quad \varphi_{z}: \gamma \mapsto \frac{\left\|z-x_{z}(\gamma)\right\|}{\gamma} .
$$

Then, the following hold:

1. $\varphi_{z}$ is nonincreasing and

$$
(\forall z \in \operatorname{dom} A) \quad \lim _{\gamma \downarrow 0^{+}} \varphi_{z}(\gamma)=\left\|\left(A+B_{1}+B_{2}\right)^{0}(z)\right\|:=\inf _{w \in\left(A+B_{1}+B_{2}\right) z}\|w\| .
$$

2. For every $\theta \in] 0,1\left[\right.$ and $z \in \operatorname{dom} B_{2}$, there exists $\gamma(z)>0$ such that, for every $\gamma \in] 0, \gamma(z)]$

$$
\left.\gamma \| B_{2} z-B_{2} x_{z}(\gamma)\right)\|\leq \theta\| z-x_{z}(\gamma) \| .
$$

Proof. Part 1: Denote $B:=B_{1}+B_{2}$. If $z \in \operatorname{zer}(A+B)$ then it follows from (2.4) that $\varphi_{z} \equiv 0$ and there is nothing to prove. Hence, assume $z \in \operatorname{dom} B_{2} \backslash \operatorname{zer}(A+$ $B$ ) which yields $\varphi_{z}(\gamma)>0$ for every $\gamma>0$. From the definition of $J_{\gamma A}$, we have $\left(z-x_{z}(\gamma)\right) / \gamma-B z \in A\left(x_{z}(\gamma)\right)$ for every $\gamma>0$ and, from the monotonicity of $A$, we deduce that, for every strictly positive constants $\gamma_{1}$ and $\gamma_{2}$ we have

$$
\begin{aligned}
0 & \leq\left\langle\frac{z-x_{z}\left(\gamma_{1}\right)}{\gamma_{1}}-\frac{z-x_{z}\left(\gamma_{2}\right)}{\gamma_{2}} \mid x_{z}\left(\gamma_{1}\right)-x_{z}\left(\gamma_{2}\right)\right\rangle \\
& =-\frac{\left\|z-x_{z}\left(\gamma_{1}\right)\right\|^{2}}{\gamma_{1}}+\left(\frac{1}{\gamma_{1}}+\frac{1}{\gamma_{2}}\right)\left\langle z-x_{z}\left(\gamma_{1}\right) \mid z-x_{z}\left(\gamma_{2}\right)\right\rangle-\frac{\left\|z-x_{z}\left(\gamma_{2}\right)\right\|^{2}}{\gamma_{2}} .
\end{aligned}
$$

Therefore

$$
\begin{aligned}
\gamma_{1} \varphi_{z}\left(\gamma_{1}\right)^{2}+\gamma_{2} \varphi_{z}\left(\gamma_{2}\right)^{2} & \leq\left(\gamma_{1}+\gamma_{2}\right)\left\langle\frac{z-x_{z}\left(\gamma_{1}\right)}{\gamma_{1}} \mid \frac{z-x_{z}\left(\gamma_{2}\right)}{\gamma_{2}}\right\rangle \\
& \leq \frac{\gamma_{1}+\gamma_{2}}{2}\left(\varphi_{z}\left(\gamma_{1}\right)^{2}+\varphi_{z}\left(\gamma_{2}\right)^{2}\right)
\end{aligned}
$$

which is equivalent to $\left(\gamma_{1}-\gamma_{2}\right)\left(\varphi_{z}\left(\gamma_{1}\right)^{2}-\varphi_{z}\left(\gamma_{2}\right)^{2}\right) \leq 0$, and the monotonicity of $\varphi_{z}$ is obtained. The limit follows from [40, Lemma 3.3\&Eq (3.5)].

Part 2: As before, if $z \in \operatorname{zer}(A+B)$ we have $z=x_{z}(\gamma)$ for every $\gamma>0$ and, hence, there is nothing to prove. From 1 we have that, for every $z \in \operatorname{dom}(A) \backslash \operatorname{zer}(A+B)$,

$$
0<\left\|z-x_{z}(1)\right\| \leq \lim _{\gamma \downarrow 0^{+}} \frac{\left\|z-x_{z}(\gamma)\right\|}{\gamma}=\left\|\left(A+B_{1}+B_{2}\right)^{0}(z)\right\| .
$$

Therefore, $\lim _{\gamma \downarrow 0^{+}} x_{z}(\gamma)=z$ and from continuity of $B_{2}, \lim _{\gamma \downarrow 0^{+}}\left\|B_{2} z-B_{2} x_{z}(\gamma)\right\|=0$. This ensures the existence of $\gamma(z)>0$ such that, for every $\gamma \in] 0, \gamma(z)]$, (2.9) holds. 
REMARK 1. Note that the previous lemma differs from [40, Lemma 3.3] because we provide the additional information $\varphi_{z}$ nonincreasing. This property is used in [4], proved in [28], and will be crucial for obtaining the convergence of the algorithm with line search to a solution to Problem 1 under weaker assumptions. We keep our proof for the sake of completeness and because the inequality (2.11) is slightly stronger than that obtained in [28].

THEOREM 2.3 (Forward-backward-half forward algorithm). Under the assumptions of Problem 1, let $z^{0} \in \operatorname{dom} A \cup X$, and consider the sequence $\left\{z_{k}\right\}_{k \in \mathbb{N}}$ recursively defined by $z^{k+1}:=T_{\gamma_{k}} z^{k}$ or, equivalently,

$$
(\forall k \in \mathbb{N}) \quad\left\lfloor\begin{array}{l}
x^{k}=J_{\gamma_{k} A}\left(z^{k}-\gamma_{k}\left(B_{1}+B_{2}\right) z^{k}\right) \\
z^{k+1}=P_{X}\left(x^{k}+\gamma_{k} B_{2} z^{k}-\gamma_{k} B_{2} x^{k}\right),
\end{array}\right.
$$

where $\left\{\gamma_{k}\right\}_{k \in \mathbb{N}}$ is a sequence of stepsizes satisfying one of the following conditions:

1. Suppose that $B_{2}$ is L-Lipschitz in $\operatorname{dom} A \cup X$. Then, for every $k \in \mathbb{N}, \gamma_{k} \in$ $[\eta, \chi-\eta]$, where $\eta \in] 0, \chi / 2[$ and $\chi$ is defined in $(2.3)$.

2. Suppose $X \subset \operatorname{dom} A$ and let $\varepsilon \in] 0,1[, \sigma \in] 0,1[$, and $\theta \in] 0, \sqrt{1-\varepsilon}[$. Then, for every $k \in \mathbb{N}, \gamma_{k}$ is the largest $\gamma \in\left\{2 \beta \varepsilon \sigma, 2 \beta \varepsilon \sigma^{2}, \cdots\right\}$ satisfying (2.9) with $z=z^{k}$, and at least one of the following additional conditions holds:

(a) $\liminf _{k \rightarrow \infty} \gamma_{k}=\delta>0$.

(b) $B_{2}$ is uniformly continuous in any weakly compact subset of $X$.

Then, $\left\{z_{k}\right\}_{k \in \mathbb{N}}$ converges weakly to a solution to Problem 1.

Proof. In the case when $B_{2}$ is $L$-Lipschitz in $\operatorname{dom} A \cup X$, it follows from Proposition 2.1(3) that the sequence $\left\{z^{k}\right\}_{k \in \mathbb{N}}$ is Fejér monotone with respect to $\operatorname{zer}\left(A+B_{1}+\right.$ $\left.B_{2}\right) \cap X$. Thus, to show that $\left\{z^{k}\right\}_{k \in \mathbb{N}}$ converges weakly to a solution to Problem 1 , we only need to prove that all of its weak subsequential limits lie in $\operatorname{zer}\left(A+B_{1}+B_{2}\right) \cap X$ [3, Theorem 5.33]. Indeed, it follows from Proposition 2.1 and our hypotheses on the stepsizes that, for every $z^{*} \in \operatorname{Fix} T_{\gamma}$,

$$
\begin{aligned}
\left\|z^{k}-z^{*}\right\|^{2}-\left\|z^{k+1}-z^{*}\right\|^{2} \geq & L^{2} \eta^{2}\left\|z^{k}-x^{k}\right\|^{2}+\frac{2 \beta \eta^{2}}{\chi}\left\|B_{1} z^{k}-B_{1} z^{*}\right\|^{2} \\
& +\frac{\chi}{2 \beta}\left\|z^{k}-x^{k}-\frac{2 \beta \gamma_{k}}{\chi}\left(B_{1} z^{k}-B_{1} z^{*}\right)\right\|^{2} .
\end{aligned}
$$

Therefore, we deduce from [15, Lemma 3.1] that

$$
z^{k}-x^{k} \rightarrow 0
$$

when $L>0$ and $0<\beta<\infty^{2}$. Now let $z \in \mathcal{H}$ be the weak limit point of some subsequence of $\left\{z^{k}\right\}_{k \in \mathbb{N}}$. Since $z^{k} \in X$ for every $k \geq 1$ and $X$ is weakly sequentially closed [3, Theorem 3.34] we deduce $z \in X$. Moreover, by denoting $B:=B_{1}+B_{2}$, it follows from $x^{k}=J_{\gamma_{k} A}\left(z^{k}-\gamma_{k} B z^{k}\right)$ that $u^{k}:=\gamma_{k}^{-1}\left(z^{k}-x^{k}\right)-B z^{k}+B x^{k} \in(A+B) x^{k}$. Then, (2.14), $\gamma_{k} \geq \eta>0$ and the Lipschitz continuity of $B$ yield $u^{k} \rightarrow 0$. Now, since $A+B_{2}$ is maximally monotone and $B_{1}$ is cocoercive with full domain, $A+B$ is maximally monotone and its graph is closed in the weak-strong topology in $\mathcal{H} \times \mathcal{H}$, which yields $0 \in A z+B z$ and the result follows.

\footnotetext{
${ }^{2}$ The case $B_{1}=0(\beta=+\infty)$ has been studied by Tseng in [40]. In the case when $B_{2}=0$ we can also obtain convergence from Proposition 2.1, since $L=0$ implies $\chi=2 \beta$ and even since the first term in the right hand side of (2.13) vanishes, the other two terms yield $z^{k}-x^{k} \rightarrow 0$.
} 
In the second case, we deduce from Proposition $2.1(1 \& 2)$ and $\gamma_{k} \leq 2 \beta \varepsilon \sigma$ that, for every $z^{*} \in \operatorname{zer}\left(A+B_{1}+B_{2}\right) \cap X$ we have

$$
\begin{aligned}
\left\|z^{k}-z^{*}\right\|^{2}-\left\|z^{k+1}-z^{*}\right\|^{2} \geq & (1-\varepsilon)\left\|z^{k}-x^{k}\right\|^{2}+\frac{\gamma_{k}}{\varepsilon}\left(2 \beta \varepsilon-\gamma_{k}\right)\left\|B_{1} z^{k}-B_{1} z^{*}\right\|^{2} \\
& +\varepsilon\left\|z^{k}-x^{k}-\frac{\gamma_{k}}{\varepsilon}\left(B_{1} z^{k}-B_{1} z^{*}\right)\right\|^{2}-\gamma_{k}^{2}\left\|B_{2} z^{k}-B_{2} x^{k}\right\|^{2} \\
\geq & \left(1-\varepsilon-\theta^{2}\right)\left\|z^{k}-x^{k}\right\|^{2}+2 \beta \varepsilon(1-\sigma) \gamma_{k}\left\|B_{1} z^{k}-B_{1} z^{*}\right\|^{2},
\end{aligned}
$$

where in the last inequality we use the conditions on $\left\{\gamma_{k}\right\}_{k \in \mathbb{N}}$, whose existence is guaranteed by Lemma $2.2(2)$ because $z^{k} \in X \subset \operatorname{dom} A$. Then, we deduce from [15, Lemma 3.1] that $z^{k}-x^{k} \rightarrow 0$. Now let $z$ be a weak limit point of a subsequence $\left\{z^{k}\right\}_{k \in K}$, with $K \subset \mathbb{N}$. If $\liminf _{k \rightarrow \infty} \gamma_{k}=\delta>0$, from (2.9) and $z^{k}-x^{k} \rightarrow 0$ we have $B_{2} x^{k}-B_{2} z^{k} \rightarrow 0$ and the proof is analogous to the previous case. Finally, for the last case, suppose that there exists a subsequence of $\left\{\gamma_{k}\right\}_{k \in K}$ (called similarly) satisfying $\lim _{k \rightarrow \infty, k \in K} \gamma_{k}=0$. Our choice of $\gamma_{k}$ yields, for every $k \in K$,

$$
\theta\left\|z^{k}-J_{\tilde{\gamma}_{k} A}\left(z^{k}-\tilde{\gamma}_{k} B z^{k}\right)\right\| / \tilde{\gamma}_{k}<\left\|B_{2} z^{k}-B_{2} J_{\tilde{\gamma}_{k} A}\left(z^{k}-\tilde{\gamma}_{k} B z^{k}\right)\right\|,
$$

where $\tilde{\gamma}_{k}=\gamma_{k} / \sigma>\gamma_{k}$ and, from Lemma 2.2(1) we have

$$
\begin{aligned}
\sigma\left\|z^{k}-J_{\tilde{\gamma}_{k} A}\left(z^{k}-\tilde{\gamma}_{k} B z^{k}\right)\right\| / \gamma_{k} & =\left\|z^{k}-J_{\tilde{\gamma}_{k} A}\left(z^{k}-\tilde{\gamma}_{k} B z^{k}\right)\right\| / \tilde{\gamma}_{k} \\
& \leq\left\|z^{k}-J_{\gamma_{k} A}\left(z^{k}-\gamma_{k} B z^{k}\right)\right\| / \gamma_{k},
\end{aligned}
$$

which, from $z^{k}-x^{k} \rightarrow 0$, yields

$$
\left\|z^{k}-J_{\tilde{\gamma}_{k} A}\left(z^{k}-\tilde{\gamma}_{k} B z^{k}\right)\right\| \leq\left\|z^{k}-x^{k}\right\| / \sigma \rightarrow 0
$$

as $k \rightarrow \infty, k \in K$. Therefore, since $z_{k} \rightarrow z$, the sequence $\left\{\tilde{x}^{k}\right\}_{k \in K}$ defined by

$$
(\forall k \in K) \quad \tilde{x}^{k}:=J_{\tilde{\gamma}_{k} A}\left(z^{k}-\tilde{\gamma}_{k} B z^{k}\right)
$$

satisfies $\tilde{x}^{k} \rightarrow z$ as $k \rightarrow+\infty, k \in K$ and

$$
\tilde{w}^{k}:=\frac{z^{k}-\tilde{x}^{k}}{\tilde{\gamma}_{k}}+B \tilde{x}^{k}-B z^{k} \in\left(A+B_{1}+B_{2}\right) \tilde{x}^{k} .
$$

Hence, since $\{z\} \cup \bigcup_{k \in \mathbb{N}}\left[\tilde{x}^{k}, z^{k}\right]$ is a weakly compact subset of $X$ [35, Lemma 3.2], it follows from the uniform continuity of $B_{2}$ that the right hand side of (2.16) goes to 0 and, hence, $\left(z^{k}-\tilde{x}^{k}\right) / \tilde{\gamma}_{k} \rightarrow 0$ as $k \rightarrow \infty, k \in K$. Moreover, since $B_{1}$ is uniformly continuous, $B=B_{1}+B_{2}$ is also locally uniformly continuous and $B \tilde{x}^{k}-B z^{k} \rightarrow 0$, which yields $\tilde{w}^{k} \rightarrow 0$ as $k \rightarrow+\infty, k \in K$. The result is obtained as in the first case since the graph of $A+B$ is weakly-strongly closed in the product topology.

REMARK 2.

1. In [40, Theorem 3.4] the local boundedness of $z \mapsto \min _{w \in(A+B) z}\|w\|$ is needed to guarantee the convergence of the method with line search. We drop this assumption by using the monotonicity of $\varphi_{z}$ in Lemma 2.2(1), which leads us to the inequality (2.17).

2. Since continuity on compact sets yields uniform continuity, in the finite dimensional setting, the assumption on $B_{2}$ reduces to the mere continuity on $X$ (see [35, Remark 3.1(v)]). In this case, we do not need to assume further assumptions than those given in Problem 1. 
REMARK 3. The maximal monotonicity assumption on $A+B_{2}$ is satisfied, for instance, if cone $\left(\operatorname{dom} A-\operatorname{dom} B_{2}\right)=\overline{\operatorname{span}}\left(\operatorname{dom} A-\operatorname{dom} B_{2}\right)$, where, for any set $D \subset \mathcal{H}$, cone $(D)=\left\{\lambda d \mid \lambda \in \mathbb{R}_{+}, d \in D\right\}$ and $\overline{\operatorname{span}}(D)$ is the smallest closed linear subspace of $\mathcal{H}$ containing $D$ [45, Theorem 3.11.11].

REMARK 4. In the case when $B_{2}$ is L-Lipschitz in $\operatorname{dom} A \cup X$, the stepsize upper bound $\chi=\chi(\beta, L)$ defined in (2.3) depends on the cocoercivity parameter $\beta$ of $B_{1}$ and the Lipschitz parameter $L$ of $B_{2}$. In order to fully recover Tseng's splitting algorithm or the forward-backward algorithm in the cases when $B_{1}$ or $B_{2}$ are zero, respectively, we study the asymptotic behaviour of $\chi(\beta, L)$ when $L \rightarrow 0$ and $\beta \rightarrow+\infty$. It is easy to verify that

$$
\lim _{L \rightarrow 0} \chi(\beta, L)=2 \beta \quad \text { and } \quad \lim _{\beta \rightarrow+\infty} \chi(\beta, L)=\frac{1}{L},
$$

which are exactly the bounds on the stepsizes of forward-backward and Tseng's splittings. On the other hand, when $B_{2}$ is continuous, if we choose $\left.\varepsilon \in\right] 0,1[$ close to 1 , $\left\{\gamma_{k}\right\}_{k \in \mathbb{N}}$ could be larger since the line search starts from $2 \beta \varepsilon \sigma$. However, $\theta<\sqrt{1-\varepsilon}$ should be close to 0 in this case, and condition (2.9) is more restrictive and satisfied only for small values of $\gamma_{k}$. Conversely, for small values of $\varepsilon$ we restrict the sequence $\left\{\gamma_{k}\right\}_{k \in \mathbb{N}}$ in a small interval but (2.9) is more easily satisfied. The optimal choice of $\varepsilon$ in order to obtain an optimal sequence $\left\{\gamma_{k}\right\}_{k \in \mathbb{N}}$ depends on the properties of the operators involved. Note that, in the particular case when $B_{2} \equiv 0,(2.9)$ is satisfied for $\theta=0$ and we can choose $\varepsilon=1$, recovering forward-backward splitting. On the other hand, when $B_{1} \equiv 0$, we can take $\varepsilon=0$ and $\left.\theta \in\right] 0,1[$ recovering the Tseng's method with backtracking proposed in [40].

3. Forward-backward-half forward splitting with non self-adjoint linear operators. In this section, we introduce modified resolvents $J_{P^{-1} A}$, which depend on an invertible linear mapping $P$. In some cases, it is preferable to compute the modified resolvent instead of the standard resolvent $J_{A}=(\mathrm{Id}+A)^{-1}$ because the former may be easier to compute than the latter or, when $P$ is triangular by blocks in a product space, the former may order the component computation of the resolvent, replacing a parallel computation with a Gauss-Seidel style sequential computation. However, $P^{-1} A$ may not be maximally monotone. The following result allows us to use some non self-adjoint linear operators in the computation of the resolvent by using specific metrics. For simplicity, we assume from here that $B_{2}$ is $L$-Lipschitz in dom $A \cup X$, for some $L \geq 0$.

Proposition 3.1. Let $A: \mathcal{H} \rightarrow 2^{\mathcal{H}}$ be a maximally monotone operator, let $P: \mathcal{H} \rightarrow \mathcal{H}$ be a linear bounded operator, and let $U:=\left(P+P^{*}\right) / 2$ and $S:=\left(P-P^{*}\right) / 2$ be the self-adjoint and skew symmetric components of $P$, respectively. Assume that there exists $\rho>0$ such that

$$
(\forall x \in \mathcal{H}) \quad \rho\|x\|^{2} \leq\langle U x \mid x\rangle=:\|x\|_{U}^{2}
$$

Then, we have

$$
J_{P^{-1} A}=J_{U^{-1}(A+S)}\left(\mathrm{Id}+U^{-1} S\right)
$$

In particular, $J_{P^{-1} A}: \mathcal{H} \rightarrow \mathcal{H}$ is single valued, everywhere defined and satisfies

$$
\left(\forall(x, y) \in \mathcal{H}^{2}\right) \quad\left\langle J_{P^{-1} A} x-J_{P^{-1} A} y \mid P x-P y\right\rangle \geq\left\|J_{P^{-1} A} x-J_{P^{-1} A} y\right\|_{U}^{2}
$$


and, hence, $U^{-1} P^{*} J_{P^{-1} A}$ is firmly nonexpansive in $\left(\mathcal{H},\langle\cdot \mid \cdot\rangle_{U}\right)$, where $\langle\cdot \mid \cdot\rangle_{U}:(x, y) \mapsto$ $\langle U x \mid y\rangle$.

Proof. Indeed, since $S$ is monotone and everywhere defined, $A+S$ is maximally monotone in $\mathcal{H}$ [3, Corollary 25.5] and, from [19, Lemma 3.7] we have that $U^{-1}(A+$ $S)$ is maximally monotone in $\mathcal{H}$ with the metric $\langle\cdot \mid \cdot\rangle_{U}:(x, y) \mapsto\langle x \mid U y\rangle$. Hence, $J_{U^{-1}(A+S)}$ is single valued (indeed firmly nonexpansive) and, for every $(x, z) \in \mathcal{H}^{2}$, we have

$$
\begin{aligned}
x=J_{U^{-1}(A+S)}\left(z+U^{-1} S z\right) & \Leftrightarrow z+U^{-1} S z-x \in U^{-1}(A+S) x \\
& \Leftrightarrow(U+S) z-(U+S) x \in A x \\
& \Leftrightarrow x=J_{P^{-1} A} z
\end{aligned}
$$

Hence, for every $(x, y) \in \mathcal{H}^{2}$, denoting by $p=J_{P^{-1} A} x=J_{U^{-1}(A+S)}\left(x+U^{-1} S x\right)$ and $q=J_{P^{-1} A} y=J_{U^{-1}(A+S)}\left(y+U^{-1} S y\right)$, the firm nonexpansivity of $J_{U^{-1}(A+S)}$ in $\left(\mathcal{H},\langle\cdot \mid \cdot\rangle_{U}\right)$ yields

$$
\begin{aligned}
\langle p-q \mid P x-P y\rangle & =\left\langle p-q \mid U\left(x+U^{-1} S x-\left(y+U^{-1} S y\right)\right)\right\rangle \\
& =\left\langle p-q \mid x+U^{-1} S x-\left(y+U^{-1} S y\right)\right\rangle_{U} \\
& \geq\|p-q\|_{U}^{2},
\end{aligned}
$$

and the result follows from $\langle p-q \mid P x-P y\rangle=\left\langle U^{-1} P^{*}(p-q) \mid x-y\right\rangle_{U}$.

Theorem 3.2 (New Metrics and $T_{\gamma}$ ). Under the hypotheses of Problem 1 and assuming additionally that $B_{2}$ is L-Lipschitz in $\operatorname{dom} A \cup X$, let $P: \mathcal{H} \rightarrow \mathcal{H}$ be a bounded linear operator, let $U:=\left(P+P^{*}\right) / 2$ and $S:=\left(P-P^{*}\right) / 2$ be the the selfadjoint and skew symmetric components of $P$, respectively. Suppose that there exists $\rho>0$ such that

$$
(\forall x \in \mathcal{H}) \quad \rho\|x\|^{2} \leq\langle U x, x\rangle \quad \text { and } \quad K^{2}<\rho\left(\rho-\frac{1}{2 \beta}\right),
$$

where $K \geq 0$ is the Lipschitz constant of $B_{2}-S$. Let $z^{0} \in \operatorname{dom} A \cup X$ and let $\left\{z^{k}\right\}_{k \in \mathbb{N}}$ be the sequence defined by the following iteration:

$$
(\forall k \in \mathbb{N}) \quad\left[\begin{array}{l}
x^{k}=J_{P^{-1} A}\left(z^{k}-P^{-1}\left(B_{1}+B_{2}\right) z^{k}\right) \\
z^{k+1}=P_{X}^{U}\left(x^{k}+U^{-1}\left(B_{2} z^{k}-B_{2} x^{k}-S\left(z^{k}-x^{k}\right)\right)\right),
\end{array}\right.
$$

where $P_{X}^{U}$ is the projection operator of $X$ under the inner product $\langle\cdot, \cdot\rangle_{U}$. Then $\left\{z^{k}\right\}_{k \in \mathbb{N}}$ converges weakly to a solution to Problem 1 .

Proof. Note that, since $U$ is invertible from (3.4), by adding and subtracting the skew term $S$, Problem 1 is equivalent to

$$
\text { find } x \in X \text { such that } 0 \in U^{-1}(A+S) x+U^{-1} B_{1} x+U^{-1}\left(B_{2}-S\right) x .
$$

Because $S$ and $-S$ are both monotone and Lipschitz, $\mathcal{A}:=U^{-1}(A+S)$ is monotone; $\mathcal{B}_{1}:=U^{-1} B_{1}$ is $\rho \beta$-cocoercive [22, Proposition 1.5]; and $\mathcal{B}_{2}:=U^{-1}\left(B_{2}-S\right)$ is monotone and $\rho^{-1} K$-Lipschitz in $\operatorname{dom} A \cup X$ under the inner product $\langle\cdot, \cdot\rangle_{U}=\langle U \cdot \mid \cdot\rangle$, where $K$ is the Lipschitz constant of $C:=B_{2}-S{ }^{3}$ For the last assertion note that, for every $x, y \in \operatorname{dom} A \cup X$,

$$
\left\|\mathcal{B}_{2} x-\mathcal{B}_{2} y\right\|_{U}^{2}=\left\langle U^{-1}(C x-C y), C x-C y\right\rangle \leq \rho^{-1} K^{2}\|x-y\|^{2} \leq \rho^{-2} K^{2}\|x-y\|_{U}^{2} .
$$

\footnotetext{
${ }^{3}$ Note that $K \leq L+\|S\|$, but this constant is not precise when, for instance, $B_{2}=S$.
} 
Moreover, the stepsize condition reduces to

$$
\gamma=1<\frac{4 \beta \rho}{1+\sqrt{1+16 \beta^{2} K^{2}}}=\frac{-\rho+\sqrt{\rho^{2}+16 \beta^{2} \rho^{2} K^{2}}}{4 \beta K^{2}}
$$

or, equivalently,

$$
\left(4 \beta K^{2}+\rho\right)^{2}<\rho^{2}+16 \beta^{2} \rho^{2} K^{2} \quad \Leftrightarrow \quad 2 \beta K^{2}+\rho<2 \beta \rho^{2},
$$

which yields the second condition in (3.4). Therefore, since $\mathcal{A}+\mathcal{B}_{2}=U^{-1}\left(A+B_{2}\right)$ is maximally monotone in $\left(\mathcal{H},\|\cdot\|_{U}\right)$, the inclusion (3.6) meets the conditions of Theorem 2.3 under this metric. Therefore, by considering the sequence generated by $z^{k+1}=T_{1} z^{k}$ for the quasi-nonexpansive operator

$$
T_{1}=P_{X}^{U} \circ\left[\left(\mathrm{Id}-\mathcal{B}_{2}\right) \circ J_{\mathcal{A}} \circ\left(\mathrm{Id}-\left(\mathcal{B}_{1}+\mathcal{B}_{2}\right)\right)+\mathcal{B}_{2}\right],
$$

which, from Proposition 3.1 reduces to (3.5), we obtain a sequence that weakly converges to a fixed point of $T_{1}$, and hence, to a solution of $\operatorname{zer}\left(A+B_{1}+B_{2}\right) \cap X$.

\section{REMARK 5.}

1. Note that, in the particular case when $P=\mathrm{Id} / \gamma$, the algorithm (3.5) reduces to (2.12) when the stepsizes are constant. Moreover, $U=P, S=0, K=L$, $\rho=1 / \gamma$ and the second condition in (3.4) reduces to $\gamma<\chi$ with $\chi$ defined in (2.3). Hence, this assumption can be seen as a kind of "step size" condition on $P$.

2. As in Remark 4, note that the second condition in (3.4) depends on the cocoercivity parameter $\beta$ and the Lipschitz constant $L$. In the case when $B_{1}$ is zero, we can take $\beta \rightarrow+\infty$ and this condition reduces to $K<\rho$. On the other hand, if $B_{2}$ is zero we can take $L=0$, then $K=\|S\|$ and, hence, the condition reduces to $\|S\|^{2}<\rho(\rho-1 /(2 \beta))$. In this way we obtain convergent versions of Tseng's splitting and forward-backward algorithm with non self-adjoint linear operators by setting $B_{1}=0$ or $B_{2}=0$ in (3.5), respectively.

3. When $S=0$ and $B_{1}=0$ or $B_{2}=0$, from Theorem 3.2 we recover the versions of Tseng's forward-backward-forward splitting [41, Theorem 3.1] or forward-backward [19, Theorem 4.1], respectively, when the step-sizes and the non-standard metrics involved are constant. Of course, when $S=0$, $U=\operatorname{Id} / \gamma$, and $\rho=1 / \gamma$, we recover the classical bound for step-sizes in the standard metric case for each method.

4. For a particular choice of operators and metric, the forward-backward method with non-standard metric discused before has been used for solving primaldual composite inclusions and primal-dual optimization problems [21, 42]. This approach generalizes, e.g., the method in [13]. In Section 5 we compare the application of our method in the primal-dual context with [42] and other methods in the literature.

5. In the particular instance when $B_{1}=B_{2}=0$, we need $\|S\|<\rho$ and we obtain from (3.5) the following version of the proximal point algorithm (we consider $X=\mathcal{H}$ for simplicity)

$$
\begin{aligned}
z^{0} \in \mathcal{H}, \quad(\forall k \in \mathbb{N}) \quad z^{k+1} & =J_{P^{-1} A} z^{k}+U^{-1} S\left(J_{P^{-1} A} z^{k}-z^{k}\right) \\
& =\left(\operatorname{Id}-U^{-1} P\right) z^{k}+U^{-1} P J_{P^{-1} A} z^{k} .
\end{aligned}
$$


Moreover, in the case when $A=B_{2}=0$, since $U^{-1} \circ S \circ P^{-1}=U^{-1}-P^{-1}$, we recover from (3.5) the gradient-type method:

$$
z^{0} \in \mathcal{H}, \quad(\forall k \in \mathbb{N}) \quad z^{k+1}=z^{k}-U^{-1} B_{1} z^{k} .
$$

6. In the particular case when $X=\mathcal{H}$ and $B_{2}$ is linear, in [30] a method involving $B_{2}^{*}$ is proposed. In the case when, $B_{2}$ is skew linear, i.e., $B_{2}^{*}=-B_{2}(3.4)$ reduces to this method in the case $\alpha_{n} \equiv 1$ and $S=P$. The methods are different in general.

4. Allowing variable $P$ and avoiding inversion of $U$. In Algorithm (3.5), the linear operator $U$ must be inverted. In this section, for the special case dom $B_{2}=X=$ $\mathcal{H}$, we show how to replace this sometimes costly inversion with a single multiplication by the map $P$, which, in addition, may vary at each iteration. This new feature is a consequence of Proposition 4.1 below, which allows us to obtain from an operator of the class $\mathfrak{T}$ in $\left(\mathcal{H},\|\cdot\|_{U}\right)$, another operator of the same class in $(\mathcal{H},\|\cdot\|)$ preserving the set of fixed points. This change to the standard metric allows us to use different linear operators at each iteration by avoiding classical restrictive additional assumptions of the type $U_{n+1} \preccurlyeq U_{n}\left(1+\eta_{n}\right)$ with $\left(\eta_{n}\right)_{n \in \mathbb{N}}$ in $\ell_{+}^{1}$. We recall that an operator $\mathcal{S}: \mathcal{H} \rightarrow \mathcal{H}$ belongs to the class $\mathfrak{T}$ in $(\mathcal{H},\|\cdot\|)$ if and only if $\operatorname{dom} \mathcal{S}=\mathcal{H}$ and $(\forall y \in \operatorname{Fix} \mathcal{S})(\forall x \in$ H) $\quad\|x-\mathcal{S} x\|^{2} \leq\langle x-\mathcal{S} x \mid x-y\rangle$.

Proposition 4.1. Let $U: \mathcal{H} \rightarrow \mathcal{H}$ be a self-adjoint bounded linear operator such that, for every $x \in \mathcal{H},\langle U x \mid x\rangle \geq \rho\|x\|^{2}$, for some $\rho>0$, let $0<\mu \leq\|U\|^{-1}$, and let $\mathcal{S}: \mathcal{H} \rightarrow \mathcal{H}$ be an operator in the class $\mathfrak{T}$ in $\left(\mathcal{H},\|\cdot\|_{U}\right)$. Then, the operator $\mathcal{Q}=\operatorname{Id}-\mu U(\operatorname{Id}-\mathcal{S})$ belongs to the class $\mathfrak{T}$ in $(\mathcal{H},\|\cdot\|)$ and $\operatorname{Fix} \mathcal{S}=\operatorname{Fix} \mathcal{Q}$.

Proof. First note that, under the assumptions on $U$ it is invertible and, from [18, Lemma 2.1], we deduce

$$
(\forall x \in \mathcal{H}) \quad\|x\|_{U}^{2}=\langle U x \mid x\rangle=\left\langle U x \mid U^{-1} U x\right\rangle \geq\|U\|^{-1}\|U x\|^{2},
$$

and $\operatorname{Fix} \mathcal{S}=\operatorname{Fix} \mathcal{Q}$ thus follows from the definition of $\mathcal{Q}$. Now let $y \in \operatorname{Fix} \mathcal{S}$ and $x \in \mathcal{H}$. We have from (4.1) that

$$
\begin{aligned}
\|x-\mathcal{S} x\|_{U}^{2} \leq\langle x-\mathcal{S} x \mid x-y\rangle_{U} & \Leftrightarrow\|x-\mathcal{S} x\|_{U}^{2} \leq\langle U(x-\mathcal{S} x) \mid x-y\rangle \\
& \Rightarrow\|U\|^{-1}\|U(x-\mathcal{S} x)\|^{2} \leq\langle U(x-\mathcal{S} x) \mid x-y\rangle \\
& \Leftrightarrow \frac{\|U\|^{-1}}{\mu}\|\mu U(x-\mathcal{S} x)\|^{2} \leq\langle\mu U(x-\mathcal{S} x) \mid x-y\rangle \\
& \Leftrightarrow \frac{\|U\|^{-1}}{\mu}\|x-\mathcal{Q} x\|^{2} \leq\langle x-\mathcal{Q} x \mid x-y\rangle
\end{aligned}
$$

and, hence, if $\left.\mu \in] 0,\|U\|^{-1}\right]$ we deduce the result.

TheOREm 4.2. Under the hypotheses of Problem 1and assuming additionally that $B_{2}$ is L-Lipschitz in $\operatorname{dom} B_{2}=\mathcal{H}$, let $\left\{P_{k}\right\}_{k \in \mathbb{N}}$ be a sequence of bounded, linear maps from $\mathcal{H}$ to $\mathcal{H}$. For each $k \in \mathbb{N}$, let $U_{k}:=\left(P_{k}+P_{k}^{*}\right) / 2$ and $S_{k}:=\left(P_{k}-P_{k}^{*}\right) / 2$ be the self-adjoint and skew symmetric components of $P_{k}$, respectively. Suppose that $M:=$ $\sup _{k \in \mathbb{N}}\left\|U_{k}\right\|<\infty$ and that there exist $\left.\varepsilon \in\right] 0,(2 M)^{-1}\left[, \rho>0\right.$, and $\left\{\rho_{k}\right\}_{k \in \mathbb{N}} \subseteq[\rho, \infty[$ such that, for every $k \in \mathbb{N}$,

$$
(\forall x \in \mathcal{H}) \quad \rho_{k}\|x\|^{2} \leq\left\langle U_{k} x, x\right\rangle \quad \text { and } \quad K_{k}^{2} \leq \frac{\rho_{k}}{1+\varepsilon}\left(\frac{\rho_{k}}{1+\varepsilon}-\frac{1}{2 \beta}\right),
$$


where $K_{k} \geq 0$ is the Lipschitz constant of $B_{2}-S_{k}$. Let $\left\{\lambda_{k}\right\}_{k \in \mathbb{N}}$ be a sequence in $\left[\varepsilon,\left\|U_{k}\right\|^{-1}-\varepsilon\right]$, let $z^{0} \in \mathcal{H}$, and let $\left\{z^{k}\right\}_{k \in \mathbb{N}}$ be a sequence of points defined by the following iteration:

$$
(\forall k \in \mathbb{N}) \quad\left[\begin{array}{l}
x^{k}=J_{P_{k}^{-1} A}\left(z^{k}-P_{k}^{-1}\left(B_{1}+B_{2}\right) z^{k}\right) \\
z^{k+1}=z^{k}+\lambda_{k}\left(P_{k}\left(x^{k}-z^{k}\right)+B_{2} z^{k}-B_{2} x^{k}\right) .
\end{array}\right.
$$

Then $\left\{z^{k}\right\}_{k \in \mathbb{N}}$ converges weakly to a solution to Problem 1 .

Proof. For every invertible and bounded linear map $P: \mathcal{H} \rightarrow \mathcal{H}$, let us denote by $\mathcal{T}_{P}: \mathcal{H} \rightarrow \mathcal{H}$ the forward-backward-forward operator of Theorem 3.2 in the case $X=\mathcal{H}$, which associates, to every $z \in \mathcal{H}$,

$$
\mathcal{T}_{P} z=x_{z}+U^{-1}\left(B_{2} z-B_{2} x_{z}-S\left(z-x_{z}\right)\right),
$$

where $x_{z}=J_{P^{-1} A}\left(z-P^{-1}\left(B_{1}+B_{2}\right) z\right)$. Recall that, from (2.2) and the proof of Theorem 3.2, $\mathcal{T}_{P}$ is a quasi-nonexpansive mapping in $\mathcal{H}$ endowed with the scalar product $\langle\cdot \mid \cdot\rangle_{U}$. Observe that multiplying Id $-\mathcal{T}_{P}$ by $U$ on the left yields a $U^{-1}$-free expression:

$$
\begin{aligned}
\left(\operatorname{Id}-\mathcal{T}_{P}\right)(z) & =\left(z-x_{z}\right)+U^{-1} S\left(z-x_{z}\right)-U^{-1}\left(B_{2} z-B_{2} x_{z}\right) \\
\Leftrightarrow \quad U\left(\operatorname{Id}-\mathcal{T}_{P}\right)(z) & =(U+S)\left(z-x_{z}\right)+B_{2} x_{z}-B_{2} z \\
& =P\left(z-x_{z}\right)+B_{2} x_{z}-B_{2} z .
\end{aligned}
$$

Note that, since $\mathcal{T}_{P}$ is quasi-nonexpansive in $\left(\mathcal{H},\|\cdot\|_{U}\right)$, it follows from [15, Proposition 2.2] that $\mathcal{S}:=\left(\operatorname{Id}+\mathcal{T}_{P}\right) / 2$ belongs to the class $\mathfrak{T}$ in $\left(\mathcal{H},\|\cdot\|_{U}\right)$ and, from Proposition 4.1 and (4.5) we obtain that the operator

$$
\mathcal{Q}_{P}:=\operatorname{Id}-\|U\|^{-1} U(\operatorname{Id}-\mathcal{S})=\operatorname{Id}-\frac{\|U\|^{-1}}{2} U\left(\operatorname{Id}-\mathcal{T}_{P}\right)
$$

belongs to the class $\mathfrak{T}$ in $(\mathcal{H},\|\cdot\|)$ and $\operatorname{Fix} \mathcal{S}=\operatorname{Fix} \mathcal{Q}_{P}=\operatorname{zer}\left(U\left(\operatorname{Id}-\mathcal{T}_{P}\right)\right)=\operatorname{Fix}\left(\mathcal{T}_{P}\right)=$ $\operatorname{zer}\left(A+B_{1}+B_{2}\right)$. Hence, from (4.5) and (4.6), the algorithm (4.4) can be written equivalently as

$$
\begin{aligned}
z^{k+1} & =z^{k}-\lambda_{k}\left(P_{k}\left(z^{k}-x_{z^{k}}\right)+B_{2} x_{z^{k}}-B_{2} z^{k}\right) \\
& =z^{k}+2 \lambda_{k}\left\|U_{k}\right\|\left(\mathcal{Q}_{P_{k}} z^{k}-z^{k}\right) .
\end{aligned}
$$

Hence, since $0<\liminf \lambda_{k}\left\|U_{k}\right\| \leq \limsup \lambda_{k}\left\|U_{k}\right\|<1$, it follows from [15, Proposition 4.2 and Theorem 4.3] that $\left(\left\|z^{k}-\mathcal{Q}_{P_{k}} z^{k}\right\|^{2}\right)_{k \in \mathbb{N}}$ is a summable sequence and $\left\{z^{k}\right\}_{k \in \mathbb{N}}$ converges weakly in $(\mathcal{H},\langle\cdot \mid \cdot\rangle)$ to a solution to $\cap_{k \in \mathbb{N}} \operatorname{Fix} \mathcal{T}_{P_{k}}=\operatorname{zer}\left(A+B_{1}+\right.$ $\left.B_{2}\right)$ if and only if every weak limit of the sequence is a solution. Note that, since (4.3) yields $\left\|U_{k}^{-1}\right\| \leq \rho_{k}^{-1}$, we have

$$
\begin{aligned}
\left\|z^{k}-\mathcal{T}_{P_{k}} z^{k}\right\|_{U_{k}}^{2} & =\left\langle U_{k}\left(z^{k}-\mathcal{T}_{P_{k}} z^{k}\right) \mid z^{k}-\mathcal{T}_{P_{k}} z^{k}\right\rangle \\
& \leq\left\|U_{k}\left(z^{k}-\mathcal{T}_{P_{k}} z^{k}\right)\right\|\left\|z^{k}-\mathcal{T}_{P_{k}} z^{k}\right\| \\
& =\left\|U_{k}^{-1}\right\|\left\|U_{k}\left(z^{k}-\mathcal{T}_{P_{k}} z^{k}\right)\right\|^{2} \\
& \leq 4\left\|U_{k}\right\|^{2} \rho_{k}^{-1}\left\|z^{k}-\mathcal{Q}_{P_{k}} z^{k}\right\|^{2} \\
& \leq 4 M^{2} \rho^{-1}\left\|z^{k}-\mathcal{Q}_{P_{k}} z^{k}\right\|^{2} \rightarrow 0 .
\end{aligned}
$$


Moreover, since $\mathcal{T}_{P_{k}}$ coincides with $T_{1}$ defined in (3.9) involving the operators $\mathcal{A}_{k}:=$ $U_{k}^{-1}\left(A+S_{k}\right), \mathcal{B}_{1, k}=U_{k}^{-1} B_{1}$, and $\mathcal{B}_{2, k}=U_{k}^{-1}\left(B_{2}-S_{k}\right)$ which are monotone, $\rho_{k} \beta$ cocoercive, and monotone and $\rho_{k}^{-1} K_{k}$-lipschitzian in $\left(\mathcal{H},\|\cdot\|_{U_{k}}\right)$, respectively, we deduce from (2.2) that, for every $z^{*} \in \operatorname{zer}\left(A+B_{1}+B_{2}\right)=\cap_{k \in \mathbb{N}} \operatorname{zer}\left(\mathcal{A}_{k}+\mathcal{B}_{1, k}+\mathcal{B}_{2, k}\right)$ we have

$$
\begin{aligned}
& \rho_{k}^{-2} K_{k}^{2}\left(\chi_{k}^{2}-1\right)\left\|z^{k}-J_{P_{k}^{-1} A}\left(z^{k}-P_{k}^{-1}\left(B_{1}+B_{2}\right) z^{k}\right)\right\|_{U_{k}}^{2} \\
& +\frac{2 \beta \rho_{k}}{\chi_{k}}\left(\chi_{k}-1\right)\left\|U_{k}^{-1}\left(B_{1} z^{k}-B_{1} z^{*}\right)\right\|_{U_{k}}^{2} \\
& +\frac{\chi_{k}}{2 \beta \rho_{k}}\left\|z^{k}-J_{P_{k}^{-1} A}\left(z^{k}-P_{k}^{-1}\left(B_{1} z^{k}+B_{2} z^{k}\right)\right)-\frac{2 \beta \rho_{k}}{\chi_{k}} U_{k}^{-1}\left(B_{1} z^{k}-B_{1} z^{*}\right)\right\|_{U_{k}}^{2} \\
& \leq\left\|z^{k}-z^{*}\right\|_{U_{k}}^{2}-\left\|\mathcal{T}_{P_{k}} z^{k}-z^{*}\right\|_{U_{k}}^{2} \\
& =-\left\|\mathcal{T}_{P_{k}} z^{k}-z^{k}\right\|_{U_{k}}^{2}-2\left\langle\mathcal{T}_{P_{k}} z^{k}-z^{k}, z^{*}-z^{k}\right\rangle_{U_{k}} \\
& \leq-\left\|\mathcal{T}_{P_{k}} z^{k}-z^{k}\right\|_{U_{k}}^{2}+2 M\left\|\mathcal{T}_{P_{k}} z^{k}-z^{k}\right\|_{U_{k}}\left\|z^{*}-z^{k}\right\|,
\end{aligned}
$$

where

$$
\chi_{k}:=\frac{4 \beta \rho_{k}}{1+\sqrt{1+16 \beta^{2} K_{k}^{2}}} \leq \rho_{k} \min \left\{2 \beta, K_{k}^{-1}\right\} .
$$

By straightforward computations in the line of (3.7) and (3.8) we deduce that (4.3) implies, for all $k \in \mathbb{N}, \chi_{k} \geq 1+\varepsilon, K_{k} \leq \rho_{k} \leq\left\|U_{k}\right\| \leq M$ and, hence, we deduce from (4.9) and (4.3) that

$$
\begin{aligned}
& \frac{\varepsilon \rho K_{k}^{2}}{M^{2}}\left\|z^{k}-J_{P_{k}^{-1} A}\left(z^{k}-P_{k}^{-1}\left(B_{1}+B_{2}\right) z^{k}\right)\right\|^{2}+\varepsilon \rho\left\|U_{k}^{-1}\left(B_{1} z^{k}-B_{1} z^{*}\right)\right\|^{2} \\
& +\frac{\rho}{2 \beta M}\left\|z^{k}-J_{P_{k}^{-1} A}\left(z^{k}-P_{k}^{-1}\left(B_{1} z^{k}+B_{2} z^{k}\right)\right)-\frac{2 \beta \rho_{k}}{\chi_{k}} U_{k}^{-1}\left(B_{1} z^{k}-B_{1} z^{*}\right)\right\|^{2} \\
& \quad \leq-\left\|\mathcal{T}_{P_{k}} z^{k}-z^{k}\right\|_{U_{k}}^{2}+2 M\left\|\mathcal{T}_{P_{k}} z^{k}-z^{k}\right\|_{U_{k}}\left\|z^{*}-z^{k}\right\| .
\end{aligned}
$$

Now, let $z$ be a weak limit of some subsequence of $\left(z^{k}\right)_{k \in \mathbb{N}}$ called similarly for simplicity. We have that $\left(\left\|z^{*}-z^{k}\right\|\right)_{k \in \mathbb{N}}$ is bounded and, since (4.8) implies $\left\|z^{k}-\mathcal{T}_{P_{k}} z^{k}\right\|_{U_{k}}^{2} \rightarrow$ 0 we deduce from (4.11) that, by denoting $x^{k}:=J_{P_{k}^{-1} A}\left(z^{k}-P_{k}^{-1}\left(B_{1}+B_{2}\right) z^{k}\right)$, that $z^{k}-x^{k} \rightarrow 0$. Hence, since, for every $x \in \mathcal{H}$,

$\left\|S_{k} x\right\| \leq\left\|\left(S_{k}-B_{2}\right) x-\left(S_{k}-B_{2}\right) 0\right\|+\left\|B_{2} x-B_{2} 0\right\| \leq\left(K_{k}+L\right)\|x\| \leq(M+L)\|x\|$,

we have

$$
\begin{aligned}
\left\|P_{k}\left(z^{k}-x^{k}\right)\right\| & =\left\|\left(U_{k}+S_{k}\right)\left(z^{k}-x^{k}\right)\right\| \\
& \leq\left\|U_{k}\left(z^{k}-x^{k}\right)\right\|+\left\|S_{k}\left(z^{k}-x^{k}\right)\right\| \\
& \leq(2 M+L)\left\|z^{k}-x^{k}\right\| \rightarrow 0 .
\end{aligned}
$$

Finally, denoting by $B:=B_{1}+B_{2}$ we have

$$
u^{k}:=P_{k}\left(z^{k}-x^{k}\right)-\left(B z^{k}-B x^{k}\right) \in(A+B) x^{k},
$$

and since $z^{k}-x^{k} \rightarrow 0$ and $B$ is continuous, it follows from (4.13) that $u^{k} \rightarrow 0$ and the result follows from the weak-strong closedness of the maximally monotone operator $A+B$ and [3, Theorem 5.33]. 
7

REMARK 6.

1. Note that, in the particular case when $S_{k} \equiv 0$ and $P_{k}=U_{k}=\gamma_{k}^{-1} V_{k}^{-1}$, we have from [18, Lemma 2.1] that $\rho_{k}=\gamma_{k}^{-1}\left\|V_{k}^{-1}\right\|$, the conditions on the constants involved in Theorem 4.2 reduce to

$$
\frac{\left\|V_{k}^{-1}\right\|}{M} \leq \gamma_{k} \leq \frac{\left\|V_{k}^{-1}\right\|}{\rho}, \quad L^{2} \leq \frac{\gamma_{k}^{-1}\left\|V_{k}^{-1}\right\|}{1+\varepsilon}\left(\frac{\gamma_{k}^{-1}\left\|V_{k}^{-1}\right\|}{1+\varepsilon}-\frac{1}{2 \beta}\right),
$$

for some $0<\rho<M$, for every $k \in \mathbb{N}$, and (4.4) reduces to

$$
(\forall k \in \mathbb{N}) \quad\left[\begin{array}{l}
x^{k}=J_{\gamma_{k} V_{k} A}\left(z^{k}-\gamma_{k} V_{k}\left(B_{1}+B_{2}\right) z^{k}\right) \\
z^{k+1}=z^{k}+\frac{\lambda_{k}}{\gamma_{k}}\left(V_{k}^{-1}\left(x^{k}-z^{k}\right)+\gamma_{k} B_{2} z^{k}-\gamma_{k} B_{2} x^{k}\right) .
\end{array}\right.
$$

If in addition we assume that $B_{2}=0$ and, hence $L=0,(4.15)$ reduces to $\gamma_{k} \leq\left\|V_{k}^{-1}\right\| 2 \beta /(1+\varepsilon)$ which is more general than the condition in [19] and, moreover, we do not need any compatibility assumption on $\left(V_{k}\right)_{k \in \mathbb{N}}$ for achieving convergence. Similarly, if $B_{1}=0$, and hence, we can take $\beta \rightarrow$ $\infty$, (4.15) reduces to $\gamma_{k} \leq\left\|V_{k}^{-1}\right\| /(L(1+\varepsilon))$ which is more general than the condition in [41] and no additional assumption on $\left(V_{k}\right)_{k \in \mathbb{N}}$ is needed. However, (4.16) involves an additional computation of $V_{k}^{-1}$ in the last step of each iteration $k \in \mathbb{N}$.

2. In the particular case when, for every $k \in \mathbb{N}, P_{k}=U_{k}=\mathrm{Id} / \gamma_{k}$, where $\left(\gamma_{k}\right)_{k \in \mathbb{N}}$ is a real sequence, we have $S_{k} \equiv 0, K_{k} \equiv L,\left\|U_{k}\right\|=\rho_{k}=1 / \gamma_{k}$, and conditions $\sup _{k \in \mathbb{N}}\left\|U_{k}\right\|<\infty$ and (4.3) reduce to

$$
0<\inf _{k \in \mathbb{N}} \gamma_{k} \leq \sup _{k \in \mathbb{N}} \gamma_{k}<\chi
$$

where $\chi$ is defined in (2.3) and (4.4) reduces to

$$
(\forall k \in \mathbb{N}) \quad\left[\begin{array}{l}
x^{k}=J_{\gamma_{k} A}\left(z^{k}-\gamma_{k}\left(B_{1}+B_{2}\right) z^{k}\right) \\
z^{k+1}=z^{k}+\eta_{k}\left(x^{k}+\gamma_{k} B_{2} z^{k}-\gamma_{k} B_{2} x^{k}-z^{k}\right),
\end{array}\right.
$$

where $\eta_{k} \in[\varepsilon, 1-\varepsilon]$, which is a relaxed version of Theorem 2.3.

3. As in Remark 2, by setting $B_{1}=0$ or $B_{2}=0$, we can derive from (4.4) versions of Tseng's splitting and forward-backward algorithm with non selfadjoint linear operators but without needing the inversion of $U$. In particular, the proximal point algorithm in (3.10) reduces to

$$
z^{0} \in \mathcal{H}, \quad(\forall k \in \mathbb{N}) \quad z^{k+1}=z^{k}+\lambda P\left(J_{P^{-1} A} z^{k}-z^{k}\right)
$$

for $\lambda<\|U\|^{-1}$ and, in the case of (3.11), to avoid inversion is to come back to the gradient-type method with the standard metric.

5. Primal-dual composite monotone inclusions with non self-adjoint linear operators. In this section, we apply our algorithm to composite primal-dual monotone inclusions involving a cocoercive and a lipschitzian monotone operator.

Problem 2. Let $\mathrm{H}$ be a real Hilbert space, let $\mathrm{X} \subset \mathrm{H}$ be closed and convex, let $z \in \mathrm{H}$, let $\mathrm{A}: \mathrm{H} \rightarrow 2^{\mathrm{H}}$ be maximally monotone, let $\mathrm{C}_{1}: \mathrm{H} \rightarrow \mathrm{H}$ be $\mu$-cocoercive, for some $\mu \in] 0,+\infty\left[\right.$, and let $\mathrm{C}_{2}: \mathrm{H} \rightarrow \mathrm{H}$ be a monotone and $\delta$-lipschitzian operator, for 
some $\delta \in] 0,+\infty\left[\right.$. Let $m \geq 1$ be an integer, and, for every $i \in\{1, \ldots, m\}$, let $\mathrm{G}_{i}$ be a real Hilbert space, let $r_{i} \in \mathrm{G}_{i}$, let $\mathrm{B}_{i}: \mathrm{G}_{i} \rightarrow 2^{\mathrm{G}_{i}}$ be maximally monotone, let $\mathrm{D}_{i}: \mathrm{G}_{i} \rightarrow$ $2^{\mathrm{G}_{i}}$ be maximally monotone and $\nu_{i}$-strongly monotone, for some $\left.\nu_{i} \in\right] 0,+\infty[$, and suppose that $\mathrm{L}_{i}: \mathrm{H} \rightarrow \mathrm{G}_{i}$ is a nonzero linear bounded operator. The problem is to solve the primal inclusion.

$$
\text { find } \mathrm{x} \in \mathrm{X} \quad \text { such that } \mathrm{z} \in \mathrm{Ax}+\sum_{i=1}^{m} \mathrm{~L}_{i}^{*}\left(\mathrm{~B}_{i} \square \mathrm{D}_{i}\right)\left(\mathrm{L}_{i} \mathrm{x}-\mathrm{r}_{i}\right)+\mathrm{C}_{1} \mathrm{x}+\mathrm{C}_{2} \mathrm{x}
$$

together with the dual inclusion

$$
\begin{aligned}
& \text { find } \mathrm{v}_{1} \in \mathrm{G}_{1}, \ldots, \mathrm{v}_{m} \in \mathrm{G}_{m} \\
& \text { such that }(\exists \mathrm{x} \in \mathrm{X})\left\{\begin{array}{l}
\mathrm{z}-\sum_{i=1}^{m} \mathrm{~L}_{i}^{*} \mathrm{v}_{i} \in \mathrm{Ax}+\mathrm{C}_{1} \mathrm{x}+\mathrm{C}_{2} \mathrm{x} \\
(\forall i \in\{1, \ldots, m\}) \mathrm{v}_{i} \in\left(\mathrm{B}_{i} \square \mathrm{D}_{i}\right)\left(\mathrm{L}_{i} \mathrm{x}-\mathrm{r}_{i}\right)
\end{array}\right.
\end{aligned}
$$

under the assumption that a solution exists.

In the case when $\mathrm{X}=\mathrm{H}$ and $\mathrm{C}_{2}=0$, Problem 2 is studied in $[42]^{4}$ and models a large class of problems including optimization problems, variational inequalities, equilibrium problems, among others (see $[8,27,42,21]$ and the references therein). In [42] the author rewrite (5.1) and (5.2) in the case $\mathrm{X}=\mathrm{H}$ as

$$
\text { find } z \in \mathcal{H} \text { such that } 0 \in M z+S z+Q z
$$

where $\mathcal{H}=\mathrm{H} \times \mathrm{G}_{1} \times \cdots \times \mathrm{G}_{m}, M: \mathcal{H} \rightarrow 2^{\mathcal{H}}:\left(\mathrm{x}, \mathrm{v}_{1}, \ldots, \mathrm{v}_{m}\right) \mapsto(\mathrm{Ax}-\mathrm{z}) \times\left(\mathrm{B}_{1}^{-1} \mathrm{v}_{1}+\right.$ $\left.\mathrm{r}_{1}\right) \times \cdots \times\left(\mathrm{B}_{m}^{-1} \mathrm{v}_{m}+\mathrm{r}_{m}\right)$ is maximally monotone, $S: \mathcal{H} \rightarrow \mathcal{H}:\left(\mathrm{x}, \mathrm{v}_{1}, \ldots, \mathrm{v}_{m}\right) \mapsto$ $\left(\sum_{i=1}^{m} \mathrm{~L}_{i}^{*} \mathrm{v}_{i},-\mathrm{L}_{1} \mathrm{x}, \ldots,-\mathrm{L}_{m} \mathrm{x}\right)$ is skew linear, and $Q: \mathcal{H} \rightarrow \mathcal{H}:\left(\mathrm{x}_{1} \mathrm{v}_{1}, \ldots, \mathrm{v}_{m}\right) \mapsto$ $\left(\mathrm{C}_{1} \mathrm{x}, \mathrm{D}_{1}^{-1} \mathrm{v}_{1}, \ldots, \mathrm{D}_{m}^{-1} \mathrm{v}_{m}\right)$ is cocoercive. If $\left(\mathrm{x}, \mathrm{v}_{1}, \ldots, \mathrm{v}_{m}\right)$ is a solution in the primaldual space $\mathcal{H}$ to $(5.3)$, then $\mathrm{x}$ is a solution to $(5.1)$ and $\left(\mathrm{v}_{1}, \ldots, \mathrm{v}_{m}\right)$ is a solution to (5.2). The author provide an algorithm for solving (5.1)-(5.2) in this particular instance, which is an application of the forward-backward splitting (FBS) applied to the inclusion

$$
\text { find } z \in \mathcal{H} \text { such that } 0 \in V^{-1}(M+S) z+V^{-1} Q z
$$

where $V$ is a specific symmetric strongly monotone operator. Under the metric $\langle V \cdot \mid \cdot\rangle$, $V^{-1}(M+S)$ is maximally monotone and $V^{-1} Q$ is cocoercive and, therefore, the FBS converges weakly to a primal-dual solution.

In order to tackle the case $\mathrm{C}_{2} \neq 0$, we propose to use the method in Theorem 4.2 for solving $0 \in A x+B_{1} x+B_{2} x$ where $A=M, B_{1}=Q, B_{2}=S+C_{2}$, and $C_{2}:\left(\mathrm{x}, \mathrm{v}_{1}, \ldots, \mathrm{v}_{m}\right) \mapsto\left(\mathrm{C}_{2} \mathrm{x}, 0, \ldots, 0\right)$ allowing, in that way, non self-adjoint linear operators which may vary among iterations. The following result provides the method thus obtained, where the dependence of the non self-adjoint linear operators with respect to iterations has been avoided for simplicity.

Theorem 5.1. In Problem 2, set $\mathrm{X}=\mathrm{H}$, set $\mathrm{G}_{0}=\mathrm{H}$, for every $i \in\{0,1, \ldots, m\}$ and $j \in\{0, \ldots, i\}$, let $\mathrm{P}_{i j}: \mathrm{G}_{j} \rightarrow \mathrm{G}_{i}$ be a linear operator satisfying

$$
\left(\forall \mathrm{x}_{i} \in \mathrm{G}_{i}\right) \quad\left\langle\mathrm{P}_{i i} \mathrm{x}_{i} \mid \mathrm{x}_{i}\right\rangle \geq \varrho_{i}\left\|\mathrm{x}_{i}\right\|^{2}
$$

\footnotetext{
${ }^{4}$ Note that in [42], weights $\left(\omega_{i}\right)_{1 \leq i \leq m}$ multiplying operators $\left(\mathrm{B}_{i} \square \mathrm{D}_{i}\right)_{1 \leq i \leq m}$ are considered. They can be retrieved in (5.1) by considering $\left(\omega_{i} \mathrm{~B}_{i}\right)_{1 \leq i \leq m}$ and $\left(\omega_{i} \mathrm{D}_{i}\right)_{1 \leq i \leq m}$ instead of $\left(\mathrm{B}_{i}\right)_{1 \leq i \leq m}$ and $\left(\mathrm{D}_{i}\right)_{1 \leq i \leq m}$. Then both formulations are equivalent.
} 
for some $\varrho_{i}>0$. Define the $(m+1) \times(m+1)$ symmetric real matrices $\Upsilon, \Sigma$, and $\Delta$ by

$$
\begin{aligned}
&(\forall i \in\{0, \ldots, m\})(\forall j<i) \quad \Upsilon_{i j}= \begin{cases}0, & \text { if } i=j ; \\
\left\|\mathrm{P}_{i j}\right\| / 2, & \text { if } i>j,\end{cases} \\
& \Sigma_{i j}= \begin{cases}\left\|\mathrm{P}_{i i}-\mathrm{P}_{i i}^{*}\right\| / 2, & \text { if } i=j ; \\
\left\|\mathrm{L}_{i}+\mathrm{P}_{i 0} / 2\right\|, & \text { if } i \geq 1 ; j=0 ; \\
\left\|\mathrm{P}_{i j}\right\| / 2, & \text { if } i>j>0,\end{cases}
\end{aligned}
$$

and $\Delta=\operatorname{Diag}\left(\varrho_{0}, \ldots, \varrho_{m}\right)$. Assume that $\Delta-\Upsilon$ is positive definite with smallest eigenvalue $\rho>0$ and that

$$
\left(\|\Sigma\|_{2}+\delta\right)^{2}<\rho\left(\rho-\frac{1}{2 \beta}\right),
$$

where $\beta=\min \left\{\mu, \nu_{1}, \ldots, \nu_{m}\right\}$. Let $M=\max _{i=0, \ldots, m}\left\|\mathrm{P}_{i i}\right\|+\|\Upsilon\|_{2}$, let $\left.\lambda \in\right] 0, M^{-1}[$, let $\left(\mathrm{x}^{0}, \mathrm{u}_{1}^{0}, \ldots, \mathrm{u}_{m}^{0}\right) \in \mathrm{H} \times \mathrm{G}_{1} \times \cdots \times \mathrm{G}_{m}$, and let $\left\{\mathrm{x}^{k}\right\}_{k \in \mathbb{N}}$ and $\left\{\mathrm{u}_{i}^{k}\right\}_{k \in \mathbb{N}, 1 \leq i \leq m}$ the sequences generated by the following routine: for every $k \in \mathbb{N}$

$$
\mid \begin{aligned}
& \mathrm{y}^{k}=J_{\mathrm{P}_{00}^{-1} \mathrm{~A}}\left(\mathrm{x}^{k}-\mathrm{P}_{00}^{-1}\left(\mathrm{C}_{1} \mathrm{x}^{k}+\mathrm{C}_{2} \mathrm{x}^{k}+\sum_{i=1}^{m} \mathrm{~L}_{i}^{*} \mathrm{u}_{i}^{k}\right)\right) \\
& \mathrm{v}_{1}^{k}=J_{\mathrm{P}_{11}^{-1} \mathrm{~B}_{1}^{-1}}\left(\mathrm{u}_{1}^{k}-\mathrm{P}_{11}^{-1}\left(\mathrm{D}_{1}^{-1} \mathrm{u}_{1}^{k}-\mathrm{L}_{1} \mathrm{x}^{k}-\mathrm{P}_{10}\left(\mathrm{x}^{k}-\mathrm{y}^{k}\right)\right)\right) \\
& \mathrm{v}_{2}^{k}=J_{\mathrm{P}_{22}^{-1} \mathrm{~B}_{2}^{-1}}\left(\mathrm{u}_{2}^{k}-\mathrm{P}_{22}^{-1}\left(\mathrm{D}_{2}^{-1} \mathrm{u}_{2}^{k}-\mathrm{L}_{2} \mathrm{x}^{k}-\mathrm{P}_{20}\left(\mathrm{x}^{k}-\mathrm{y}^{k}\right)-\mathrm{P}_{21}\left(\mathrm{u}_{1}^{k}-\mathrm{v}_{1}^{k}\right)\right)\right) \\
& \vdots \\
& \mathrm{v}_{m}^{k}=J_{\mathrm{P}_{m m}^{-1} \mathrm{~B}_{m}^{-1}}\left(\mathrm{u}_{m}^{k}-\mathrm{P}_{m m}^{-1}\left(\mathrm{D}_{m}^{-1} \mathrm{u}_{m}^{k}-\mathrm{L}_{m} \mathrm{x}^{k}-\mathrm{P}_{m 0}\left(\mathrm{x}^{k}-\mathrm{y}^{k}\right)-\sum_{j=1}^{m-1} \mathrm{P}_{m j}\left(\mathrm{u}_{j}^{k}-\mathrm{v}_{j}^{k}\right)\right)\right) \\
& \mathrm{x}^{k+1}=\mathrm{x}^{k}+\lambda\left(\mathrm{P}_{00}\left(\mathrm{y}^{k}-\mathrm{x}^{k}\right)+\left(\mathrm{C}_{2} \mathrm{x}^{k}-\mathrm{C}_{2} \mathrm{y}^{k}+\sum_{i=1}^{m} \mathrm{~L}_{i}^{*}\left(\mathrm{u}_{i}^{k}-\mathrm{v}_{i}^{k}\right)\right)\right) \\
& \mathrm{u}_{1}^{k+1}=\mathrm{u}_{1}^{k}+\lambda\left(\mathrm{P}_{10}\left(\mathrm{y}^{k}-\mathrm{x}^{k}\right)+\mathrm{P}_{11}\left(\mathrm{v}_{1}^{k}-\mathrm{u}_{1}^{k}\right)-\mathrm{L}_{1}\left(\mathrm{x}^{k}-\mathrm{y}^{k}\right)\right) \\
& \vdots \\
& \mathrm{u}_{m}^{k+1}=\mathrm{u}_{m}^{k}+\lambda\left(\mathrm{P}_{m 0}\left(\mathrm{y}^{k}-\mathrm{x}^{k}\right)+\sum_{j=1}^{m} \mathrm{P}_{m j}\left(\mathrm{v}_{j}^{k}-\mathrm{u}_{j}^{k}\right)-\mathrm{L}_{m}\left(\mathrm{x}^{k}-\mathrm{y}^{k}\right)\right)
\end{aligned}
$$

Then there exists a primal-dual solution $\left(\mathrm{x}^{*}, \mathrm{u}_{1}^{*}, \ldots, \mathrm{u}_{m}^{*}\right) \in \mathrm{H} \times \mathrm{G}_{1} \times \cdots \times \mathrm{G}_{m}$ to Problem 2 such that $\mathrm{x}^{k} \rightarrow \mathrm{x}^{*}$ and, for every $i \in\{1, \ldots, m\}, \mathrm{u}_{i}^{k} \rightarrow \mathrm{u}_{i}^{*}$.

Proof. Consider the real Hilbert space $\mathcal{H}=\mathrm{H} \oplus \mathrm{G}_{1} \oplus \cdots \oplus \mathrm{G}_{m}$, where its scalar product and norm are denoted by $\langle\langle\cdot \mid \cdot\rangle\rangle$ and $\||\cdot|\| \mid$, respectively, and $x=$ $\left(\mathrm{x}_{0}, \mathrm{x}_{1}, \ldots, \mathrm{x}_{m}\right)$ and $y=\left(\mathrm{y}_{0}, \mathrm{y}_{1}, \ldots, \mathrm{y}_{m}\right)$ denote generic elements of $\mathcal{H}$. Similarly as in [42], note that the set of primal-dual solutions $x^{*}=\left(\mathrm{x}^{*}, \mathrm{u}_{1}^{*}, \ldots, \mathrm{u}_{m}^{*}\right) \in \mathcal{H}$ to Problem 2 in the case $\mathrm{X}=\mathrm{H}$ coincides with the set of solutions to the monotone inclusion

$$
\text { find } \quad x \in \mathcal{H} \text { such that } 0 \in A x+B_{1} x+B_{2} x
$$

where the operators $A: \mathcal{H} \rightarrow 2^{\mathcal{H}}, B_{1}: \mathcal{H} \rightarrow \mathcal{H}$, and $B_{2}: \mathcal{H} \rightarrow \mathcal{H}\left(\operatorname{dom} B_{2}=\mathcal{H}\right)$ defined by

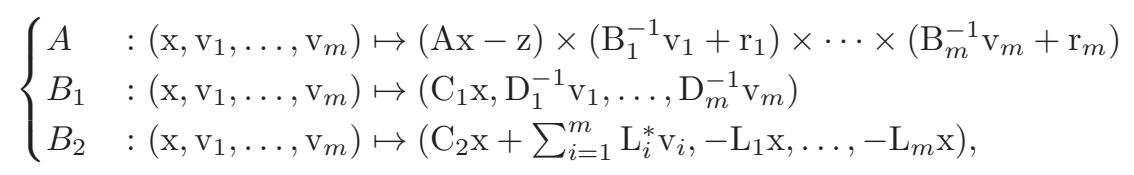


are maximally monotone, $\beta$-cocoercive, and monotone-Lipschitz, respectively (see $[3$, Proposition 20.22 and 20.23] and [42, Eq. (3.12)]).

Now let $P: \mathcal{H} \rightarrow \mathcal{H}$ defined by

$$
P: x \mapsto\left(\mathrm{P}_{00} \mathrm{x}_{0}, \mathrm{P}_{10} \mathrm{x}_{0}+\mathrm{P}_{11} \mathrm{x}_{1}, \ldots, \sum_{j=0}^{m} \mathrm{P}_{m j} \mathrm{x}_{j}\right)=\left(\sum_{j=0}^{i} \mathrm{P}_{i j} \mathrm{x}_{j}\right)_{i=0}^{m} .
$$

Then $P^{*}: x \mapsto\left(\sum_{j=i}^{m} \mathrm{P}_{j i}^{*} \mathrm{x}_{j}\right)_{i=0}^{m}$ and $U: \mathcal{H} \rightarrow \mathcal{H}$ and $S: \mathcal{H} \rightarrow \mathcal{H}$ defined by

$$
\begin{aligned}
& U: x \mapsto\left(\frac{1}{2} \sum_{j=0}^{i-1} \mathrm{P}_{i j} \mathrm{x}_{j}+\left(\frac{\mathrm{P}_{i i}+\mathrm{P}_{i i}^{*}}{2}\right) \mathrm{x}_{i}+\frac{1}{2} \sum_{j=i+1}^{m} \mathrm{P}_{j i}^{*} \mathrm{x}_{j}\right)_{i=0}^{m} \\
& S: x \mapsto\left(\frac{1}{2} \sum_{j=0}^{i-1} \mathrm{P}_{i j} \mathrm{x}_{j}+\left(\frac{\mathrm{P}_{i i}-\mathrm{P}_{i i}^{*}}{2}\right) \mathrm{x}_{i}-\frac{1}{2} \sum_{j=i+1}^{m} \mathrm{P}_{j i}^{*} \mathrm{x}_{j}\right)_{i=0}^{m}
\end{aligned}
$$

are the self-adjoint and skew components of $P$, respectively, satisfying $P=U+S$. Moreover, for every $x=\left(\mathrm{x}_{0}, \mathrm{x}_{1}, \ldots, \mathrm{x}_{m}\right)$ in $\mathcal{H}$, we have

$$
\begin{aligned}
\langle\langle U x \mid x\rangle\rangle & =\sum_{i=0}^{m} \frac{1}{2} \sum_{j=0}^{i-1}\left\langle\mathrm{P}_{i j} \mathrm{x}_{j} \mid \mathrm{x}_{i}\right\rangle+\left\langle\mathrm{P}_{i i} \mathrm{x}_{i} \mid \mathrm{x}_{i}\right\rangle+\frac{1}{2} \sum_{j=i+1}^{m}\left\langle\mathrm{P}_{j i}^{*} \mathrm{x}_{j} \mid \mathrm{x}_{i}\right\rangle \\
& =\sum_{i=0}^{m}\left\langle\mathrm{P}_{i i} \mathrm{x}_{i} \mid \mathrm{x}_{i}\right\rangle+\sum_{i=1}^{m} \sum_{j=0}^{i-1}\left\langle\mathrm{P}_{i j} \mathrm{x}_{j} \mid \mathrm{x}_{i}\right\rangle \\
& \geq \sum_{i=0}^{m} \varrho\left\|\mathrm{x}_{i}\right\|^{2}-\sum_{i=1}^{m} \sum_{j=0}^{i-1}\left\|\mathrm{P}_{i j}\right\|\left\|\mathrm{x}_{i}\right\|\left\|\mathrm{x}_{j}\right\| \\
& =\xi \cdot(\Delta-\Upsilon) \xi \geq \rho|\xi|^{2}=\rho\|\mid\| x \|^{2},
\end{aligned}
$$

where $\xi:=\left(\left\|\mathrm{x}_{i}\right\|\right)_{i=0}^{m} \in \mathbb{R}^{m+1}, \Upsilon$ is defined in (5.6), and $\rho$ is the smallest (strictly positive) eigenvalue of $\Delta-\Upsilon$. In addition, we can write $B_{2}-S=C_{2}+R$, where $C_{2}: x \mapsto\left(\mathrm{C}_{2} \mathrm{x}, 0, \ldots, 0\right)$ is monotone and $\delta$-lipschitzian, and $R$ is a skew linear operator satisfying, for every $x=\left(\mathrm{x}_{0}, \mathrm{x}_{1}, \ldots, \mathrm{x}_{m}\right) \in \mathcal{H}, R x=\left(\sum_{j=0}^{m} R_{i, j} \mathrm{x}_{j}\right)_{0 \leq i \leq m}$, where the operators $R_{i, j}: \mathrm{G}_{j} \rightarrow \mathrm{G}_{i}$ are defined by $R_{i, j}=-\mathrm{P}_{i j} / 2$ if $i>j>0, R_{i, j}=$ $-\left(\mathrm{L}_{i}+\mathrm{P}_{i 0}\right) / 2$ if $i>j=0, R_{i, i}=\left(\mathrm{P}_{i i}^{*}-\mathrm{P}_{i i}\right) / 2$ and the other components follow from the skew property of $R$. Therefore,

$\left\||| R x\left|\left\|^{2}=\sum_{i=0}^{m}\right\| \sum_{j=0}^{m} R_{i, j} \mathrm{x}_{j}\left\|^{2} \leq \sum_{i=0}^{m}\left(\sum_{j=0}^{m}\left\|R_{i, j}\right\|\left\|\mathrm{x}_{j}\right\|\right)^{2}=|\Sigma \xi|^{2} \leq\right\| \Sigma\left\|_{2}^{2}|\xi|^{2}=\right\| \Sigma\left\|_{2}^{2} \mid\right\| x \|^{2}\right.\right.$,

from which we obtain that $B_{2}-S$ is $\left(\delta+\|\Sigma\|_{2}\right)$-lipschitzian. Altogether, by noting that, for every $x \in \mathcal{H},\|U x\| \leq M$, all the hypotheses of Theorem 4.2 hold in this instance and by developing (4.4) for this specific choices of $A, B_{1}, B_{2}, P, \gamma$, and setting, for every $k \in \mathbb{N}, z^{k}=\left(\mathrm{x}^{k}, \mathrm{u}_{1}^{k}, \ldots, \mathrm{u}_{m}^{k}\right)$ and $x^{k}=\left(\mathrm{y}^{k}, \mathrm{v}_{1}^{k}, \ldots, \mathrm{v}_{m}^{k}\right)$, we obtain (5.8) after straighforward computations and using

$$
x^{k}=J_{P^{-1} A}\left(z^{k}-P^{-1}\left(B_{1} z^{k}+B_{2} z^{k}\right)\right) \quad \Leftrightarrow \quad P\left(z^{k}-x^{k}\right)-\left(B_{1} z^{k}+B_{2} z^{k}\right) \in A x^{k} .
$$


The result follows, hence, as a consequence of Theorem 4.2.

\section{REMARK 7.}

1. As in Theorem 4.2, the algorithm in Theorem 5.1 allows for linear operators $\left(\mathrm{P}_{i j}\right)_{0 \leq i, j \leq m}$ depending on the iteration, whenever (4.3) holds for the corresponding operators defined in (5.11)-(5.13). We omit this generalization in Theorem 5.1 for the sake of simplicity.

2. In the particular case when, for every $i \in\{1, \ldots, m\}, B_{i}=\widetilde{B}_{i} \square M_{i}$, where $M_{i}$ is such that $M_{i}^{-1}$ is monotone and $\sigma_{i}$-Lipschitz, for some $\sigma_{i}>0$, Problem (2) can be solved in a similar way if, instead of $B_{2}$ and $\delta$, we consider $\widetilde{B}_{2}:\left(\mathrm{x}, \mathrm{v}_{1}, \ldots, \mathrm{v}_{m}\right) \mapsto\left(\mathrm{C}_{2} \mathrm{x}+\sum_{i=1}^{m} \mathrm{~L}_{i}^{*} \mathrm{v}_{i}, \mathrm{M}_{1}^{-1} \mathrm{v}_{1}-\mathrm{L}_{1} \mathrm{x}, \ldots, \mathrm{M}_{m}^{-1} \mathrm{v}_{m}-\mathrm{L}_{m} \mathrm{x}\right)$ and $\widetilde{\delta}=\max \left\{\delta, \sigma_{1}, \ldots, \sigma_{m}\right\}$. Again, for the sake of simplicity, this extension has not been considered in Problem 2.

3. If the inversion of the matrix $U$ is not difficult or no variable metric is used and the projection onto $\mathrm{X} \subset \mathrm{H}$ is computable, we can also use Theorem 3.2 for solving Problem 2 in the general case $\mathrm{X} \subset \mathrm{H}$.

Corollary 5.2. In Problem 2, let $\theta \in[-1,1]$, let $\sigma_{0}, \ldots, \sigma_{m}$ be strictly positive real numbers and let $\Omega$ the $(m+1) \times(m+1)$ symmetric real matrix given by

$$
(\forall i, j \in\{0, \ldots, m\}) \quad \Omega_{i j}= \begin{cases}\frac{1}{\sigma_{i}}, & \text { if } i=j \\ -\left(\frac{1+\theta}{2}\right)\left\|\mathrm{L}_{i}\right\|, & \text { if } 0=j<i \\ 0, & \text { if } 0<j<i .\end{cases}
$$

Assume that $\Omega$ is positive definite with $\rho>0$ its smallest eigenvalue and that

$$
\left(\delta+\left(\frac{1-\theta}{2}\right) \sqrt{\sum_{i=1}^{m}\left\|\mathrm{~L}_{i}\right\|^{2}}\right)^{2}<\rho\left(\rho-\frac{1}{2 \beta}\right)
$$

where $\beta=\min \left\{\mu, \nu_{1}, \ldots, \nu_{m}\right\}$. Let $M=\left(\min \left\{\sigma_{0}, \ldots, \sigma_{m}\right\}\right)^{-1}+\left(\frac{1+\theta}{2}\right) \sqrt{\sum_{i=1}^{m}\left\|\mathrm{~L}_{i}\right\|^{2}}$, let $\lambda \in] 0, M^{-1}\left[\right.$, let $\left(\mathrm{x}^{0}, \mathrm{u}_{1}^{0}, \ldots, \mathrm{u}_{m}^{0}\right) \in \mathrm{H} \times \mathrm{G}_{1} \times \cdots \times \mathrm{G}_{m}$, and let $\left\{\mathrm{x}^{k}\right\}_{k \in \mathbb{N}}$ and $\left\{\mathrm{u}_{i}^{k}\right\}_{k \in \mathbb{N}, 1 \leq i \leq m}$ the sequences generated by the following routine:

$(\forall k \in \mathbb{N})$

$$
\begin{aligned}
& \mathrm{y}^{k}=J_{\sigma_{0} \mathrm{~A}}\left(\mathrm{x}^{k}-\sigma_{0}\left(\mathrm{C}_{1} \mathrm{x}^{k}+\mathrm{C}_{2} \mathrm{x}^{k}+\sum_{i=1}^{m} \mathrm{~L}_{i}^{*} \mathrm{u}_{i}^{k}\right)\right) \\
& \text { For every } i=1, \ldots, m \\
& \left\lfloor\mathrm{v}_{i}^{k}=J_{\sigma_{i} \mathrm{~B}_{i}^{-1}}\left(\mathrm{u}_{i}^{k}-\sigma_{i}\left(\mathrm{D}_{i}^{-1} \mathrm{u}_{i}^{k}-\mathrm{L}_{i}\left(\mathrm{y}^{k}+\theta\left(\mathrm{y}^{k}-\mathrm{x}^{k}\right)\right)\right)\right)\right. \\
& \mathrm{x}^{k+1}=\mathrm{x}^{k}+\frac{\lambda}{\sigma_{0}}\left(\mathrm{y}^{k}-\mathrm{x}^{k}+\sigma_{0}\left(\mathrm{C}_{2} \mathrm{x}^{k}-\mathrm{C}_{2} \mathrm{y}^{k}+\sum_{i=1}^{m} \mathrm{~L}_{i}^{*}\left(\mathrm{u}_{i}^{k}-\mathrm{v}_{i}^{k}\right)\right)\right) \\
& \text { For every } i=1, \ldots, m \\
& \left\lfloor\mathrm{u}_{i}^{k+1}=\mathrm{u}_{i}^{k}+\frac{\lambda}{\sigma_{i}}\left(\mathrm{v}_{i}^{k}-\mathrm{u}_{i}^{k}-\sigma_{i} \theta \mathrm{L}_{i}\left(\mathrm{y}^{k}-\mathrm{x}^{k}\right)\right)\right.
\end{aligned}
$$

Then there exists a primal-dual solution $\left(\mathrm{x}^{*}, \mathrm{u}_{1}^{*}, \ldots, \mathrm{u}_{m}^{*}\right) \in \mathrm{H} \times \mathrm{G}_{1} \times \cdots \times \mathrm{G}_{m}$ to Problem 2 such that $\mathrm{x}^{k} \rightarrow \mathrm{x}^{*}$ and, for every $i \in\{1, \ldots, m\}, \mathrm{u}_{i}^{k} \rightarrow \mathrm{u}_{i}^{*}$.

Proof. This result is a consequence of Theorem 5.1 when, for every $i \in\{0, \ldots, m\}$, $\mathrm{P}_{i i}=\mathrm{Id} / \sigma_{i}, \mathrm{P}_{i 0}=-(1+\theta) \mathrm{L}_{i}$, and, for every $0<j<i, \mathrm{P}_{i j}=0$. Indeed, we have from (5.5) that $\varrho_{i}=1 / \sigma_{i}$, and from (5.6) we deduce that, for every $x=\left(\xi_{i}\right)_{0 \leq i \leq m} \in \mathbb{R}^{m+1}$,

$$
\|\Sigma x\|^{2}=\left(\frac{1-\theta}{2}\right)^{2}\left[\left(\sum_{i=0}^{m}\left\|\mathrm{~L}_{i}\right\| \xi_{i}\right)^{2}+\xi_{0}^{2} \sum_{i=1}^{m}\left\|\mathrm{~L}_{i}\right\|^{2}\right] \leq\left(\frac{1-\theta}{2}\right)^{2}\left(\sum_{i=0}^{m}\left\|\mathrm{~L}_{i}\right\|^{2}\right)\|x\|^{2},
$$


from which we obtain $\|\Sigma\|_{2} \leq\left(\frac{1-\theta}{2}\right) \sqrt{\sum_{i=1}^{m}\left\|\mathrm{~L}_{i}\right\|^{2}}$. Actually, we have the equality by choosing $\bar{x}=\left(\bar{\xi}_{i}\right)_{0 \leq i \leq m}$ defined by $\bar{\xi}_{i}=\left\|\mathrm{L}_{i}\right\| / \sqrt{\sum_{j=1}^{m}\left\|\mathrm{~L}_{j}\right\|^{2}}$ for every $i \in\{1, \ldots, m\}$ and $\bar{\xi}_{0}=0$, which satisfies $\|\bar{x}\|=1$ and $\|\Sigma \bar{x}\|=\left(\frac{1-\theta}{2}\right) \sqrt{\sum_{i=1}^{m}\left\|\mathrm{~L}_{i}\right\|^{2}}$. Therefore, condition (5.7) reduces to (5.18). On the other hand, from (5.6) we deduce that $\Omega=\Delta-\Upsilon$ and $\Upsilon=\left(\frac{1+\theta}{1-\theta}\right) \Sigma$, which yields $\|\Upsilon\|_{2}=\left(\frac{1+\theta}{2}\right) \sqrt{\sum_{i=1}^{m}\left\|\mathrm{~L}_{i}\right\|^{2}}$ and $\max _{i=0, \ldots, m}\left\|\mathrm{P}_{i i}\right\|=\left(\min \left\{\sigma_{0}, \ldots, \sigma_{m}\right\}\right)^{-1}$. Altogether, since (5.19) is exactly (5.8) for this choice of matrices $\left(\mathrm{P}_{i, j}\right)_{0 \leq i, j, \leq m}$, the result is a consequence of Theorem 5.1.

REMARK 8.

1. Note that, the condition $\rho>0$ where $\rho$ is the smallest eigenvalue of $\Omega$ defined in (5.17), is guaranteed if $\sigma_{0}\left(\frac{1+\theta}{2}\right)^{2} \sum_{i=1}^{m} \sigma_{i}\left\|\mathrm{~L}_{i}\right\|^{2}<1$. Indeed, by repeating the procedure in [42, (3.20)] in finite dimension we obtain, for every $x=$ $\left(\xi_{i}\right)_{0 \leq i \leq m} \in \mathbb{R}^{m+1}$,

$$
\begin{aligned}
x \cdot \Omega x & =\sum_{i=0}^{m} \frac{\xi_{i}^{2}}{\sigma_{i}}-\sum_{i=1}^{m} 2\left(\frac{1+\theta}{2}\right) \xi_{0}\left\|\mathrm{~L}_{i}\right\| \xi_{i} \\
& =\sum_{i=0}^{m} \frac{\xi_{i}^{2}}{\sigma_{i}}-\left(\frac{1+\theta}{2}\right) \sum_{i=1}^{m} 2 \frac{\sqrt{\sigma_{i}\left\|\mathrm{~L}_{i}\right\| \xi_{0}}}{\left(\sigma_{0} \sum_{j=1}^{m} \sigma_{j}\left\|\mathrm{~L}_{j}\right\|^{2}\right)^{1 / 4}} \frac{\left(\sigma_{0} \sum_{j=1}^{m} \sigma_{j}\left\|\mathrm{~L}_{j}\right\|^{2}\right)^{1 / 4} \xi_{i}}{\sqrt{\sigma_{i}}} \\
& \geq \sum_{i=0}^{m} \frac{\xi_{i}^{2}}{\sigma_{i}}-\left(\frac{1+\theta}{2}\right)\left(\frac{\xi_{0}^{2}}{\sqrt{\sigma_{0}}} \sqrt{\sum_{j=1}^{m} \sigma_{j}\left\|\mathrm{~L}_{j}\right\|^{2}}+\sqrt{\sigma_{0} \sum_{j=1}^{m} \sigma_{j}\left\|\mathrm{~L}_{j}\right\|^{2}} \sum_{j=1}^{m} \frac{\xi_{j}^{2}}{\sigma_{j}}\right) \\
& =\left(1-\left(\frac{1+\theta}{2}\right) \sqrt{\sigma_{0} \sum_{j=1}^{m} \sigma_{j}\left\|\mathrm{~L}_{j}\right\|^{2}}\right) \sum_{i=0}^{m} \frac{\xi_{i}^{2}}{\sigma_{i}} \\
& \geq \rho_{v}\|x\|^{2}
\end{aligned}
$$

with

$$
\rho_{v}=\max \left\{\sigma_{0}, \ldots, \sigma_{m}\right\}^{-1}\left(1-\left(\frac{1+\theta}{2}\right) \sqrt{\sigma_{0} \sum_{j=1}^{m} \sigma_{j}\left\|\mathrm{~L}_{j}\right\|^{2}}\right) .
$$

Note that $\rho_{v}$ coincides with the constant obtained in [42] in the case $\theta=1$ and we have $\rho \geq \rho_{v}$. Moreover, $\sigma_{0}\left(\frac{1+\theta}{2}\right)^{2} \sum_{i=1}^{m} \sigma_{i}\left\|\mathrm{~L}_{i}\right\|^{2}<1$ is also necessary for obtaining $\rho>0$, since in (5.21) we can choose a particular vector $x$ for obtaining the equality. Of course, this choice does not guarantee to also have equality in the last inequality in (5.22) and, hence, $\rho \geq \rho_{v}$ in general.

2. If we set $\theta=1$ and $\mathrm{C}_{2}=0$ and, hence, $\delta=0$, (5.18) reduces to $2 \beta \rho>1$ and we obtain from (5.19) a variant of [42, Theorem 3.1] including an extra forward step involving only the operators $\left(\mathrm{L}_{i}\right)_{1 \leq i \leq m}$. However, our condition is less restrictive, since $\rho \geq \rho_{v}$, where $\rho_{v}$ is defined in (5.23) and it is obtained in [42] as we have seen in the last remark. Actually, in the particular case when $m=1, \mathrm{~L}_{1}=\alpha \mathrm{Id}, \sigma_{0}=\eta^{2} \sigma_{1}=: \eta \sigma$ for some $0<\eta<1$, constants $\rho_{v}$ and $\rho$ reduce to

$$
\rho_{v}(\eta)=\frac{1-\eta \sigma \alpha}{\sigma} \quad \text { and } \quad \rho(\eta)=\frac{1}{2 \sigma}\left(\frac{\eta^{2}+1}{\eta^{2}}-\sqrt{\left(\frac{\eta^{2}-1}{\eta^{2}}\right)^{2}+4 \alpha^{2} \sigma^{2}}\right)
$$


respectively. By straightforward computations we deduce that $\rho(\eta)>\rho_{v}(\eta)$ for every $0<\eta<(\alpha \sigma)^{-1}$, and hence our constant can strictly improve the condition $2 \beta \rho>1$, needed in both approaches. Moreover, since Theorem 5.1 allows for non self-adjoint linear operators varying among iterations, we can permit variable stepsizes $\sigma_{0}^{k}, \ldots, \sigma_{m}^{k}$ in Theorem 5.1, which could not be used in [42] because of the variable metric framework.

3. In the particular case when $\mathrm{C}_{1}=0$ and $\mathrm{C}_{2}=0$ we can take $\beta \rightarrow+\infty$ and, hence, condition (5.18) reduces to

$$
\left(\frac{1-\theta}{2}\right) \sqrt{\sum_{i=1}^{m}\left\|\mathrm{~L}_{i}\right\|^{2}}<\rho
$$

which is stronger than the condition in [27] for the case $m=1$, in which it is only needed that $\rho>0$ for achieving convergence. Indeed, in the case $m=1$, (5.24) reduces to $2-2 \theta \sigma_{0} \sigma_{1}\left\|\mathrm{~L}_{1}\right\|^{2}>(1-\theta)\left(\sigma_{0}+\sigma_{1}\right)\left\|\mathrm{L}_{1}\right\|$, which coincides with the condition in [27] in the case $\theta=1$, but they differ if $\theta \neq 1$ because of the extra forward step coming from the Tseng's splitting framework. Actually, in the case $\theta=0$ it reduces to $\sigma_{0}+\sigma_{1}<2 /\left\|\mathrm{L}_{1}\right\|$ and in the case $\theta=-1$ we obtain the stronger condition $\max \left\{\sigma_{0}, \sigma_{1}\right\}<1 /\left\|\mathrm{L}_{1}\right\|$. Anyway, in our context we can use constants $\sigma_{0}^{k}, \ldots, \sigma_{m}^{k}$ varying among iterations and we have a variant of the method in [27] and, in the case when $\theta=1$, of Chambolle-Pock's splitting [13].

4. Since $\rho_{v}$ defined in (5.23) satisfies $\rho_{v} \leq \rho$ in the case when $\mathrm{C}_{1}=\mathrm{C}_{2}=0$, a sufficient condition for guaranteeing (5.24) is $(1-\theta) \sqrt{\sum_{i=1}^{m}\left\|\mathrm{~L}_{i}\right\|^{2}} / 2<\rho_{v}$, which implied by the condition

$$
\max \left\{\sigma_{0}, \ldots, \sigma_{m}\right\} \sqrt{\sum_{i=1}^{m}\left\|\mathrm{~L}_{i}\right\|^{2}}<1
$$

5. Consider the case of composite optimization problems, i.e., when $\mathrm{A}=\partial \mathrm{f}$, $\mathrm{C}_{1}=\nabla \mathrm{h}$ for every $i=1, \ldots, m, \mathrm{~B}_{i}=\partial \mathrm{g}_{i}$ and $\mathrm{D}_{i}=\partial \ell_{i}$, where, for every $i=1, \ldots, m, \mathrm{f}: \mathrm{H} \rightarrow]-\infty,+\infty]$ and $\left.\left.\mathrm{g}_{i}: \mathrm{G}_{i} \rightarrow\right]-\infty,+\infty\right]$ are proper lower semicontinuous and convex functions and $\mathrm{h}: \mathrm{H} \rightarrow \mathbb{R}$ is differentiable, convex, with $\beta^{-1}$-Lipschitz gradient. In this case, any solution to Problem 2 when $\mathrm{C}_{2}=0$ is a solution to the primal-dual optimization problems

$$
\min _{\mathrm{x} \in \mathrm{H}} \mathrm{f}(\mathrm{x})+\mathrm{h}(\mathrm{x})+\sum_{i=1}^{m}\left(\mathrm{~g}_{i} \square \ell_{i}\right)\left(\mathrm{L}_{i} \mathrm{x}\right)
$$

and

$$
\min _{\mathrm{u}_{1} \in \mathrm{G}_{1}, \ldots, \mathrm{u}_{m} \in \mathrm{G}_{m}}\left(\mathrm{f}^{*} \square \mathrm{h}^{*}\right)\left(-\sum_{i=1}^{m} \mathrm{~L}_{i}^{*} \mathrm{u}_{i}\right)+\sum_{i=1}^{m} \mathrm{~g}_{i}^{*}\left(\mathrm{u}_{i}\right)+\ell_{i}^{*}\left(\mathrm{u}_{i}\right),
$$

and the equivalence holds under some qualification condition. In this partic- 
ular case, (5.19) reduces to

$$
\mid \begin{aligned}
& \mathrm{y}^{k}=\operatorname{prox}_{\sigma_{0} \mathrm{f}}\left(\mathrm{x}^{k}-\sigma_{0}\left(\nabla \mathrm{h}\left(\mathrm{x}^{k}\right)+\sum_{i=1}^{m} \mathrm{~L}_{i}^{*} \mathrm{u}_{i}^{k}\right)\right) \\
& \text { For every } i=1, \ldots, m \\
& \left\lfloor\mathrm{v}_{i}^{k}=\operatorname{prox}_{\sigma_{i} \mathrm{~g}_{i}^{*}}\left(\mathrm{u}_{i}^{k}-\sigma_{i}\left(\nabla \ell_{i}^{*}\left(\mathrm{u}_{i}^{k}\right)-\mathrm{L}_{i}\left(\mathrm{y}^{k}+\theta\left(\mathrm{y}^{k}-\mathrm{x}^{k}\right)\right)\right)\right)\right. \\
& \mathrm{x}^{k+1}=\mathrm{x}^{k}+\frac{\lambda}{\sigma_{0}}\left(\mathrm{y}^{k}-\mathrm{x}^{k}+\sigma_{0} \sum_{i=1}^{m} \mathrm{~L}_{i}^{*}\left(\mathrm{u}_{i}^{k}-\mathrm{v}_{i}^{k}\right)\right) \\
& \text { For every } i=1, \ldots, m \\
& \left\lfloor\mathrm{u}_{i}^{k+1}=\mathrm{u}_{i}^{k}+\frac{\lambda}{\sigma_{i}}\left(\mathrm{v}_{i}^{k}-\mathrm{u}_{i}^{k}-\sigma_{i} \theta \mathrm{L}_{i}\left(\mathrm{y}^{k}-\mathrm{x}^{k}\right)\right)\right.
\end{aligned}
$$

which, in the case $m=1$, is very similar to the method proposed in [30, Algorithm 3] (by taking $\mu=(1-\theta)^{-1}$ for $\theta \in[-1,0]$ ), with a slightly different choice of the parameters involved in the last two lines in (5.28). On the other hand, in the case when $\ell=0$ and $\theta=1$, it differs from [21, Algorithm 5.1] in the last two steps, in which linear operators are involved in our case. An advantage of our method, even in the case $m=1$, is that the stepsizes $\sigma_{0}$ and $\sigma_{1}$ may vary among iterations.

6. Applications. In this section we explore four applications for illustrating the advantages and flexibility of the methods proposed in the previous sections. In the first application, we apply Theorem 2.3 to the obstacle problem in PDE's in which dropping the extra forward step decreases the computational cost per iteration because the computation of an extra gradient step is numerically expensive. In the second application, devoted to empirical risk minimization (ERM), we illustrate the flexibility of using non self-adjoint linear operators. We derive different sequential algorithms depending on the nature of the linear operator involved. In the third application, we develop a distributed operator-splitting scheme which allows for time-varying communication graphs. Finally, the last application focuses in nonlinear constrained optimization, in which monotone non-Lipschitz operators arise naturally.

6.1. Obstacle problem. The obstacle problem is to find the equilibrium position of an elastic membrane on a domain $\Omega$, whose boundary is fixed and is restricted to remain above the some obstacle, given by the function $\varphi: \Omega \rightarrow \mathbb{R}$. This problem can be applied to fluid filtration in porous media, elasto-plasticity, optimal control among other disciplines (see, e.g., [11] and the references therein). Let $u: \Omega \rightarrow \mathbb{R}$ be a function representing the vertical displacement of the membrane and let $\psi: \Gamma \rightarrow \mathbb{R}$ be the function representing the fixed boundary, where $\Gamma$ is the smooth boundary of $\Omega$. Assume that $\psi \in H^{1 / 2}(\Gamma)$ and $\varphi \in C^{1,1}(\Omega)$ satisfy $\mathrm{T} \varphi \leq \psi$, and consider the problem

$$
\begin{array}{r}
\min _{\mathrm{u} \in H^{1}(\Omega)} \frac{1}{2} \int_{\Omega}|\nabla \mathrm{u}|^{2} \mathrm{dx} \\
\text { s.t. } \mathrm{Tu}=\psi, \quad \text { a.e. on } \Gamma ; \\
\mathrm{u} \geq \varphi, \quad \text { a.e. in } \Omega,
\end{array}
$$

where $\mathrm{T}: H^{1}(\Omega) \rightarrow H^{1 / 2}(\Gamma)$ is the (linear) trace operator and $H^{1}(\Omega)$ is endowed with the scalar product $\langle\cdot \mid \cdot\rangle:(\mathrm{u}, \mathrm{v}) \mapsto \int_{\Omega} \mathrm{uv} d x+\int_{\Omega} \nabla \mathrm{u} \cdot \nabla \mathrm{v} d x$. There is a unique solution to this obstacle problem [12].

In order to set this problem in our context, let us define the operator

$$
Q: H^{-1}(\Omega) \times H^{-1 / 2}(\Gamma) \rightarrow H^{1}(\Omega)
$$


which associates to each $(\mathrm{q}, \mathrm{w}) \in H^{-1}(\Omega) \times H^{-1 / 2}(\Gamma)$ the unique weak solution (in the sense of distributions) to [44, Section 25]

$$
\begin{cases}-\Delta \mathrm{u}+\mathrm{u}=\mathrm{q}, & \text { in } \Omega \\ \frac{\partial \mathrm{u}}{\partial \nu}=\mathrm{w}, & \text { on } \Gamma\end{cases}
$$

where $\nu$ is outer unit vector normal to $\Gamma$. Hence, $Q$ satisfies

$$
(\forall \mathrm{v} \in \mathrm{H}) \quad\langle Q(\mathrm{q}, \mathrm{w}) \mid \mathrm{v}\rangle=\langle\mathrm{w} \mid \mathrm{Tv}\rangle_{-1 / 2,1 / 2}+\langle\mathrm{q} \mid \mathrm{v}\rangle_{-1,1}
$$

where $\langle\cdot \mid \cdot\rangle_{-1 / 2,1 / 2}$ and $\langle\cdot \mid \cdot\rangle_{-1,1}$ stand for the dual pairs $H^{-1 / 2}(\Gamma)-H^{1 / 2}(\Gamma)$ and $H^{-1}(\Omega)-H^{1}(\Omega)$, respectively. Then, by defining $\mathrm{H}=H^{1}(\Omega), \mathrm{G}=H^{1 / 2}(\Gamma), \mathrm{f}: \mathrm{u} \mapsto$ $\frac{1}{2} \int_{\Omega}|\nabla \mathrm{u}|^{2} \mathrm{dx}, \mathrm{g}=\iota_{\mathrm{C}}$, where $\mathrm{C}=\{\mathrm{u} \in \mathrm{H} \mid \mathrm{u} \geq \varphi$ a.e. in $\Omega\}$, let $\mathrm{D}=\{\psi\}$, and let $\mathrm{L}=\mathrm{T}$, (6.1) can be written equivalently as

$$
\min _{L u \in D} f(u)+g(u) .
$$

Moreover, it is easy to verify that $\mathrm{f}$ is convex and, by using integration by parts and (6.4), for every $\mathrm{h} \in \mathrm{H}$ we have

$$
\begin{aligned}
\mathrm{f}(\mathrm{u}+\mathrm{h})-\mathrm{f}(\mathrm{u})-\left\langle Q\left(-\Delta \mathrm{u}, \frac{\partial \mathrm{u}}{\partial \nu}\right) \mid \mathrm{h}\right\rangle= & \frac{1}{2} \int_{\Omega}|\nabla \mathrm{h}|^{2} d x+\int_{\Omega} \nabla \mathrm{u} \cdot \nabla \mathrm{h} d x+\langle\Delta \mathrm{u} \mid \mathrm{h}\rangle_{-1,1} \\
& -\left\langle\frac{\partial \mathrm{u}}{\partial \nu} \mid \mathrm{Th}\right\rangle_{-1 / 2,1 / 2} \\
= & \frac{1}{2} \int_{\Omega}|\nabla \mathrm{h}|^{2} d x
\end{aligned}
$$

which yields

$$
\lim _{\|\mathrm{h}\| \rightarrow 0} \frac{\left|\mathrm{f}(\mathrm{u}+\mathrm{h})-\mathrm{f}(\mathrm{u})-\left\langle Q\left(-\Delta \mathrm{u}, \frac{\partial \mathrm{u}}{\partial \nu}\right) \mid \mathrm{h}\right\rangle\right|}{\|\mathrm{h}\|}=\frac{1}{2} \lim _{\|\mathrm{h}\| \rightarrow 0} \frac{\|\nabla \mathrm{h}\|_{L^{2}}^{2}}{\|\mathrm{~h}\|}=0 .
$$

Hence, $\mathrm{f}$ is Fréchet differentiable with a linear gradient given by $\nabla \mathrm{f}: \mathrm{u} \mapsto Q\left(-\Delta \mathrm{u}, \frac{\partial \mathrm{u}}{\partial \nu}\right)$. Moreover, from integration by parts we have

$$
\left\langle Q\left(-\Delta \mathrm{u}, \frac{\partial \mathrm{u}}{\partial \nu}\right) \mid \mathrm{h}\right\rangle=\left\langle\frac{\partial \mathrm{u}}{\partial \nu} \mid \mathrm{Th}\right\rangle_{-1 / 2,1 / 2}-\langle\Delta \mathrm{u} \mid \mathrm{h}\rangle_{-1,1}=\int_{\Omega} \nabla \mathrm{u} \cdot \nabla \mathrm{h} d x \leq\|\mathrm{u}\|\|\mathrm{h}\|,
$$

which yields $\|\nabla \mathrm{f}(\mathrm{u})\| \leq\|\mathrm{u}\|$ and, hence, it is 1-cocoercive [1]. In addition, the trace operator is linear and bounded [26] and we have from (6.4) that

$$
(\forall \mathrm{v} \in \mathrm{H})\left(\forall \mathrm{w} \in H^{1 / 2}(\Gamma)\right) \quad\langle Q(0, \mathrm{w}) \mid \mathrm{v}\rangle=\langle\mathrm{w} \mid \mathrm{Tv}\rangle_{-1 / 2,1 / 2}
$$

which yields $\mathrm{L}^{*}: \mathrm{w} \mapsto \mathrm{Q}(0, \mathrm{w})$ and since $\mathrm{C}$ is non-empty closed convex, $\mathrm{g}$ is convex, proper, lower semicontinuous and $\operatorname{prox}_{\gamma \mathrm{g}}=P_{\mathrm{C}}$, for any $\gamma>0$.

Since first order conditions of (6.5) reduce to find $(\mathrm{u}, \mathrm{w}) \in \mathrm{H} \times \mathrm{G}$ such that $0 \in N_{\mathrm{C}}(\mathrm{u})+\nabla \mathrm{f}(\mathrm{u})+\mathrm{T}^{*} N_{\mathrm{D}}(\mathrm{Tu})$, which is a particular case of Problem 2 and from 
Corollary 5.2 when $\theta=1$ the method

$$
\left[\begin{array}{l}
\mathrm{v}^{k}=P_{\mathrm{C}}\left(\mathrm{u}^{k}-\sigma_{0} Q\left(-\Delta \mathrm{u}^{k}, \frac{\partial \mathrm{u}^{k}}{\partial \nu}+\mathrm{w}^{k}\right)\right) \\
\mathrm{t}^{k}=\mathrm{w}^{k}+\sigma_{1}\left(\mathrm{~T}\left(2 \mathrm{y}^{k}-\mathrm{x}^{k}\right)-\psi\right) \\
\mathrm{u}^{k+1}=\mathrm{u}^{k}+\frac{\lambda}{\sigma_{0}}\left(\mathrm{v}^{k}-\mathrm{u}^{k}+\sigma_{0} Q\left(0, \mathrm{w}^{k}-\mathrm{t}^{k}\right)\right) \\
\mathrm{w}^{k+1}=\mathrm{w}^{k}+\frac{\lambda}{\sigma_{1}}\left(\mathrm{t}^{k}-\mathrm{w}^{k}-\sigma_{1} \mathrm{~T}\left(\mathrm{v}^{k}-\mathrm{u}^{k}\right)\right)
\end{array}\right.
$$

generates a weakly convergent sequence $\left(\mathrm{u}^{k}\right)_{k \in \mathbb{N}}$ to the unique solution to the obstacle problem provided, for instance (see Remark 8.1), that $\max \left\{\sigma_{0}, \sigma_{1}\right\}+2 \sqrt{\sigma_{0} \sigma_{1}}\|\mathrm{~T}\|<2$. Note that $\nabla \mathrm{f}$ must be computed only once at each iteration, improving the performance with respect to primal-dual methods following Tseng's approach, in which $\nabla f$ must be computed twice by iteration (see, e.g., $[8,40]$ ). The method proposed in $[21,42]$ can also solve this problem but with stronger conditions on constants $\sigma_{0}$ and $\sigma_{1}$ as studied in Remark 8. Moreover, our approach may include variable stepsizes together with different assymetric linear operators which may improve the performance of the method.

On the other hand, the general version of our method in Theorem 3.2 allows for an additional projection onto a closed convex set. In this case this can be useful to impose some of the constraints of the problem in order to guarantee that iterates at each iteration satisfy such constraints. An additional projection step may accelerate the method as it has been studied in [9]. Numerical comparisons among these methods are part of further research.

6.2. An Incremental Algorithm for Nonsmooth Empirical Risk Minimization. In machine learning [37], the Empirical Risk Minimization (ERM) problem seeks to minimize a finite sample approximation of an expected loss, under conditions on the feasible set and the loss function. If the solution to the sample approximation converges to a minimizer of the expected loss when the size of the sample increases, we say that the problem is learnable. Suppose that we have a sample of size $m$, and, for every $i \in\{1, \ldots, m\}$, the loss function associated to the sample $\mathrm{z}_{i}$ is given by $l\left(\cdot ; \mathrm{z}_{i}\right): \mathrm{x} \mapsto \mathrm{f}_{i}\left(\mathrm{a}_{i}^{\top} x\right)$, where each $\mathrm{a}_{i} \in \mathbb{R}^{d} \backslash\{0\}$ and each $\mathrm{f}_{i}: \mathbb{R} \rightarrow(-\infty, \infty]$ is closed, proper, and convex. Then the ERM problem is to

$$
\underset{\mathrm{x} \in \mathbb{R}^{d}}{\operatorname{minimize}} \frac{1}{m} \sum_{i=1}^{m} \mathrm{f}_{i}\left(\mathrm{a}_{i}^{\top} \mathrm{x}\right)
$$

This form features in support vector machines, logistic regression, linear regression, least-absolute deviations, and many other common models in machine learning.

The parameter $m$ indicates the size of the training set and is typically large. Parallelizing a (sub)gradient computation of (6.11) is straightforward, but in general, because training sets are large, we may not have enough processors to do so. Thus, when only a few processors are available, incremental iterative algorithms, in which one or a few training samples are used per iteration to update our solution estimate, are a natural choice.

Several incremental algorithms are available for solving (6.11), including incremental (sub)gradient descent and incremental aggregated gradient methods [36, 24, $29,23,6,43,5,33,7]$. The former class requires diminishing stepsizes (e.g., of size $\left.O\left(k^{-1 / 2}\right)\right)$ and, hence, their convergence may be very slow, while the latter class of 
algorithms is usually restricted to the cases in which either $f_{i}$ is smooth or the dual problem of (6.11) is smooth (in which case (6.11) is strongly convex). In contrast, we now develop an incremental proximal algorithm, which imposes no smoothness or strong convexity assumptions. It has a Gauss-Seidel structure and is obtained by an application of Theorem 5.1. The involved stepsizes may vary among iterations but they are set to be constants for simplicity.

The method follows from the following first-order optimality conditions obtained assuming some qualification condition:

$$
\mathrm{x} \quad \text { solves }(6.11) \Leftrightarrow 0 \in \sum_{i=1}^{m} \mathrm{a}_{i} \partial \mathrm{f}_{i}\left(\mathrm{a}_{i}^{\top} \mathrm{x}\right),
$$

which is a particular case of Problem 2 when $\mathrm{H}=\mathbb{R}^{d}, \mathrm{~A} \equiv\{0\}, \mathrm{C}_{1}=\mathrm{C}_{2} \equiv 0$ and, for every $i \in\{1, \ldots, m\}, \mathrm{G}_{i}=\mathbb{R}, \mathrm{D}_{i}^{-1}=0, \mathrm{~L}_{i}=\mathrm{a}_{i}^{\top}$, and $\mathrm{B}_{i}=\partial \mathrm{f}_{i}$. By using Theorem 5.1 in this case for matrices $\left(\mathrm{P}_{i j}\right)_{0 \leq i<j \leq m}$ given by

$$
(\forall 0 \leq j<i \leq m) \quad \mathrm{P}_{i j}= \begin{cases}\frac{\mathrm{Id}}{\sigma_{0}}, & \text { if } i=j=0 \\ \frac{1}{\sigma_{i}}, & \text { if } i=j>0 \\ -\mathrm{a}_{i}^{\top}, & \text { if } j=0 \\ \sigma_{0} \mathrm{a}_{i}^{\top} \mathrm{a}_{j}, & \text { if } 0<j<i\end{cases}
$$

we obtain

$$
\mid \begin{aligned}
& \mathrm{v}_{1}^{k}=\operatorname{prox}_{\sigma_{1} \mathrm{f}_{1}^{*}}\left(\mathrm{u}_{1}^{k}+\sigma_{1}\left(\mathrm{a}_{1}^{\top} \mathrm{x}^{k}-\sigma_{0} \sum_{i=1}^{m} \mathrm{a}_{1}^{\top} \mathrm{a}_{i} \mathrm{u}_{i}^{k}\right)\right) \\
& \mathrm{v}_{2}^{k}=\operatorname{prox}_{\sigma_{2} \mathrm{f}_{2}^{*}}\left(\mathrm{u}_{2}^{k}+\sigma_{2}\left(\mathrm{a}_{2}^{\top} \mathrm{x}^{k}-\sigma_{0}\left(\mathrm{a}_{2}^{\top} \mathrm{a}_{1} \mathrm{v}_{1}^{k}+\sum_{i=2}^{m} \mathrm{a}_{2}^{\top} \mathrm{a}_{i} \mathrm{u}_{i}^{k}\right)\right)\right) \\
& \quad \vdots \\
& \mathrm{v}_{m}^{k}=\operatorname{prox}_{\sigma_{m} \mathrm{f}_{m}^{*}}\left(\mathrm{u}_{m}^{k}+\sigma_{m}\left(\mathrm{a}_{m}^{\top} \mathrm{x}^{k}-\sigma_{0}\left(\sum_{i=1}^{m-1} \mathrm{a}_{m}^{\top} \mathrm{a}_{i} \mathrm{v}_{i}^{k}+\left\|\mathrm{a}_{m}\right\|^{2} \mathrm{u}_{m}^{k}\right)\right)\right) \\
& \mathrm{x}^{k+1}=\mathrm{x}^{k}-\lambda \sum_{i=1}^{m} \mathrm{a}_{i} \mathrm{v}_{i}^{k} \\
& \mathrm{u}_{1}^{k+1}=\mathrm{u}_{1}^{k}+\frac{\lambda}{\sigma_{1}}\left(\mathrm{v}_{1}^{k}-\mathrm{u}_{1}^{k}\right) \\
& \quad \vdots \\
& \mathrm{u}_{m}^{k+1}=\mathrm{u}_{m}^{k}+\frac{\lambda}{\sigma_{m}}\left(\mathrm{v}_{m}^{k}-\mathrm{u}_{m}^{k}\right)+\sigma_{0} \sum_{j=1}^{m-1} \mathrm{a}_{m}^{\top} \mathrm{a}_{j}\left(\mathrm{v}_{j}^{k}-\mathrm{u}_{j}^{k}\right) .
\end{aligned}
$$

Since conditions (5.5)-(5.7) hold if

$$
\sqrt{\sum_{i=1}^{m}\left\|\mathrm{a}_{i}\right\|^{2}}+\sigma_{0} \sum_{i=1}^{m}\left\|\mathrm{a}_{i}\right\|^{2}+\frac{\sigma_{0}}{2}\left(\max _{i=1, \ldots, m}\left\|\mathrm{a}_{i}\right\|^{2}-\min _{i=1, \ldots, m}\left\|\mathrm{a}_{i}\right\|^{2}\right)<\frac{1}{\max _{i=0, \ldots, m} \sigma_{i}}
$$

by choosing $\left(\sigma_{i}\right)_{0 \leq i \leq m}$ satisfying $(6.15)$ the sequence $\left(\mathrm{x}^{k}\right)_{k \in \mathbb{N}}$ generated by (6.14) converges to a solution provided that $\lambda<M^{-1}$ where

$$
M=\left(\min _{i=0, \ldots, m} \sigma_{i}\right)^{-1}+\frac{1}{2} \sqrt{\sum_{i=1}^{m}\left\|\mathrm{a}_{i}\right\|^{2}}+\frac{\sigma_{0}}{2}\left(\sum_{i=1}^{m}\left\|\mathrm{a}_{i}\right\|^{2}+\max _{i=1, \ldots, m}\left\|\mathrm{a}_{i}\right\|^{2}\right) .
$$

Note that, without loss of generality, we can assume, for every $i \in\{1, \ldots, m\}$, $\left\|\mathrm{a}_{i}\right\|=1$, since $\mathrm{f}_{i}\left(\mathrm{a}_{i}^{\top} \mathrm{x}\right)=\mathrm{g}_{i}\left(\left(\mathrm{a}_{i} /\left\|\mathrm{a}_{i}\right\|\right)^{\top} \mathrm{x}\right)$ with $\mathrm{g}_{i}: \mathrm{x} \mapsto \mathrm{f}_{i}\left(\left\|\mathrm{a}_{i}\right\| \mathrm{x}\right)$ and $\operatorname{prox}_{\mathrm{g}_{i}}: \mathrm{x} \mapsto$ $\operatorname{prox}_{\left\|\mathrm{a}_{i}\right\|^{2} \mathrm{f}_{i}}\left(\left\|\mathrm{a}_{i}\right\| \mathrm{x}\right) /\left\|\mathrm{a}_{i}\right\|$. Therefore, condition (6.15) can be reduced to $\sqrt{m}+m \sigma_{0}<$ $\left(\max _{i=0, \ldots, m} \sigma_{i}\right)^{-1}$, which, in the case $\sigma_{0}=\cdots=\sigma_{m}$ reduces to $\sigma_{0}<(\sqrt{5}-1) /(2 \sqrt{m})$. 
6.3. A Distributed Operator Splitting Scheme with Time-Varying Networks. In this section we develop an extension of the popular distributed operator splitting scheme $P G$-Extra $[38,39]$ to time-varying graphs. The problem data are a collection of cost functions $f_{1}, \ldots, f_{n}$ on a Hilbert space $\mathcal{H}$ and a sequence of connected, undirected communication graphs $G_{t}=\left(V_{t}, E_{t}\right)$ with vertices $V_{t}=\{1, \ldots, n\}$ and edges $E_{t} \subseteq\{1, \ldots, n\}^{2}$. Then the goal of distributed optimization is to

$$
\underset{x \in \mathcal{H}}{\operatorname{minimize}} \sum_{i=1}^{n} f_{i}(x)
$$

through an iterative algorithm that, at every time $t \in \mathbb{N}$, only allows communication between neighbors in $G_{t}$. For simplicity, we focus on the case wherein $f_{i}: \mathcal{H} \rightarrow$ ]$-\infty,+\infty]$ is proper, lower semicontinuous and convex.

A well-known distributed operator splitting schemes is known as PG-Extra. This method applies to fixed communicated graphs $G_{t} \equiv G$, and can be viewed as an instance of modern primal-dual algorithms, such as Condat- $\mathrm{Vu}[21,42]$. To the best of our knowledge there is no known extension of $P G$-Extra to time-varying graphs that may also be applied to monotone inclusions. We will now develop such an extension.

For the graph $G_{t}$, let $A_{t}$ denote its adjacency matrix and let $D_{t}$ denote its degree matrix. ${ }^{5}$ The Laplacian matrix of $G_{t}$ is defined as the difference

$$
L_{t}:=D_{t}-A_{t} .
$$

It is well-known that, for fully connected graphs, we have the identity $\operatorname{ker}\left(L_{t}\right)=$ $\operatorname{span}\left(\mathbf{1}_{n}\right)$ [14]. We may exploit this fact to develop an equivalent formulation of (6.16).

The Laplacian operator has a natural extension to the product space $\mathcal{H}^{n}$. It is then a straightforward exercise to show that the extension induces the following identity:

$$
\left(\forall \mathbf{x}:=\left(x_{1}, \ldots, x_{n}\right) \in \mathcal{H}^{n}\right) \quad L_{t} \mathbf{x}=0 \Longleftrightarrow x_{1}=x_{2}=\cdots=x_{n} .
$$

Therefore, a family of equivalent formulations of (6.16) is given by

$$
\underset{\mathbf{x} \in \mathcal{H}^{n}}{\operatorname{minimize}} \sum_{i \in V} f_{i}\left(x_{i}\right)
$$

subject to: $L_{t} \mathbf{x}=0$.

The constraint $L_{t} \mathbf{x}=0$ is equivalent to the constraint $x \in \mathcal{U}:=\left\{x \in \mathcal{H}^{n} \mid x_{1}=\ldots=\right.$ $\left.x_{n}\right\}$. Thus, one could apply a splitting method to derive a distributed algorithm consisting of decoupled proximal steps on the $f_{i}$ followed by global averaging steps induced by the projection onto $\mathcal{U}$. However, in order to develop an algorithm that respects the local communication structure of the graphs $G_{t}$, we must avoid computing such projections onto $\mathcal{U}$. For any fixed $t$, we may develop such a method as a special case of modern primal-dual algorithms.

Indeed, a straightforward application of Condat- $\mathrm{Vu}[21,42]$ yields the update rule

For all $i \in V$ in parallel

$$
\begin{aligned}
x_{i}^{k+1} & =\operatorname{prox}_{\gamma f_{i}}\left(x_{i}^{k}-\gamma\left(L_{t} \mathbf{y}^{k}\right)_{i}\right) \\
\mathbf{y}^{k+1} & =\mathbf{y}^{k}+\tau L_{t}\left(2 \mathbf{x}^{k+1}-\mathbf{x}^{k}\right),
\end{aligned}
$$

\footnotetext{
${ }^{5}$ Briefly, $\left(A_{t}\right)_{i j}=1$ if $(i, j) \in E$ and is zero otherwise, while $D$ is a diagonal matrix with diagonal entries $D_{i i}=\operatorname{deg}(i)$.
} 
where $\gamma, \tau>0$ are appropriately chosen stepsizes. This algorithm is fully decentralized because multiplications by $L_{t}$ only induce communication among neighbors in the graph $G_{t}$.

If we allow $t=k$, this Condat- $\mathrm{Vu}[21,42]$ algorithm has, to the best of our knoweldge, no supporting convergence theory, although each of the optimization problems (6.17) have the same set of solutions. The lack of convergence theory arises because Condat-Vu measures convergence in the product space $\left(\mathcal{H}^{n} \times \mathcal{H}^{n},\|\cdot\|_{P_{t}}\right)$, where $P_{t}$ is a metric inducing linear transformation depending on $L_{t}$ :

$$
P_{t}:=\left[\begin{array}{ll}
\frac{1}{\gamma} \mathrm{Id} & -L_{t} \\
-L_{t} & \frac{1}{\tau} \mathrm{Id}
\end{array}\right]
$$

One may hope to apply standard variable metric operator-splitting schemes [19, 41], but the compatibility condition cannot hope to be satisfied. Thus, instead of Condat$\mathrm{Vu}$, we apply the variable metric technique developed in this manuscript.

Mathematically, we let

$$
\begin{aligned}
\mathcal{S}_{t}: \mathcal{H}^{n} \times \mathcal{H}^{n} & \rightarrow \mathcal{H}^{n} \times \mathcal{H}^{n} \\
(\mathbf{x}, \mathbf{y}) & \mapsto\left(\left(\operatorname{prox}_{\gamma f_{i}}\left(x_{i}^{k}-\gamma\left(L_{t} y^{k}\right)_{i}\right)\right)_{i=1}^{n}, \mathbf{y}^{k}+\tau L_{t}\left(2 \mathbf{x}^{k+1}-\mathbf{x}^{k}\right)\right) .
\end{aligned}
$$

Given a proper choice of $\gamma$ and $\tau$, the results of $[21,42]$ show that $\mathcal{S}_{t}$ is of $\mathfrak{T}$-class in the space $\left(\mathcal{H}^{n} \times \mathcal{H}^{n},\|\cdot\|_{P_{t}}\right)$ (indeed, $\mathcal{S}_{t}$ is a resolvent). Thus, for any $0<\mu \leq\left\|P_{t}\right\|^{-1}$, Proposition 4.1 implies that

$$
\mathcal{Q}_{t}=\operatorname{Id}-\mu P_{t}\left(\operatorname{Id}-\mathcal{S}_{t}\right),
$$

is of $\mathfrak{T}$-class in the space $\left(\mathcal{H}^{n} \times \mathcal{H}^{n},\|\cdot\|\right)$ and $\operatorname{Fix}\left(\mathcal{Q}_{t}\right)=\operatorname{Fix}\left(\mathcal{S}_{t}\right)$. Like $\mathcal{S}_{t}$, the operator $\mathcal{Q}_{t}$ may be computed in a decentralized fashion, as communication between agents is only induced through multiplications by $L_{t}$.

The algorithm resulting from applying $Q_{t}$ is a time-varying distributed operatorsplitting scheme:

$$
\left(\mathbf{x}^{k+1}, \mathbf{y}^{k+1}\right)=\mathcal{Q}_{k}\left(\mathbf{x}^{k}, \mathbf{y}^{k}\right)
$$

The convergence of this iteration may be proved using an argument similar to Theorem 4.2 (which does not capture the case in which the operator at hand is varying). To prove convergence of this iteration, one must observe that the $\operatorname{Fix}\left(\mathcal{Q}_{k}\right)$ is constant, that for all $\left(\mathbf{x}^{*}, \mathbf{y}^{*}\right) \in \operatorname{Fix}\left(Q_{k}\right)$ the sequence $\|\left(\left(\mathbf{x}^{k}, \mathbf{y}^{k}\right)-\left(\mathbf{x}^{*}, \mathbf{y}^{*}\right) \|\right.$ is nonincreasing, and that $\sum_{k=0}^{\infty}\left\|\left(\mathbf{x}^{k+1}, \mathbf{y}^{k+1}\right)-\left(\mathbf{x}^{k}, \mathbf{y}^{k}\right)\right\|^{2}<\infty$. A standard argument then shows that $\left(\mathbf{x}^{k}, \mathbf{y}^{k}\right)$ converges to an element of $\operatorname{Fix}\left(\mathcal{Q}_{k}\right) \equiv \operatorname{Fix}\left(\mathcal{Q}_{0}\right)$.

6.4. Nonlinear constrained optimization problems. In this application we aim at solving the nonlinear constrained optimization problem

$$
\underset{x \in C}{\operatorname{minimize}} f(x)+h(x),
$$

where $\left.\left.C=\left\{x \in \mathcal{H} \mid(\forall i \in\{1, \ldots, p\}) \quad g_{i}(x) \leq 0\right\}, f: \mathcal{H} \rightarrow\right]-\infty,+\infty\right]$ is lower semicontinuous, convex and proper, for every $i \in\{1, \ldots, p\}, g_{i}: \operatorname{dom}\left(g_{i}\right) \subset \mathcal{H} \rightarrow \mathbb{R}$ and $h: \mathcal{H} \rightarrow \mathbb{R}$ are $\mathcal{C}^{1}$ convex functions in int dom $g_{i}$ and $\mathcal{H}$, respectively, and $\nabla h$ is $\beta^{-1}$-Lipschitz. A solution of the optimization problem (6.19) can be found via the saddle points of the Lagrangian

$$
L(x, u)=f(x)+h(x)+u^{\top} g(x)-\iota_{\mathbb{R}_{+}^{p}}(u),
$$


which, under standard qualification conditions can be found by solving the monotone inclusion (see [34])

$$
\text { find } x \in Y \quad \text { such that }\left(\exists u \in \mathbb{R}_{+}^{p}\right) \quad(0,0) \in A(x, u)+B_{1}(x, u)+B_{2}(x, u),
$$

where $Y \subset \mathcal{H}$ is a nonempty closed convex set modeling apriori information on the solution (eventually we can take $Y=\mathcal{H}), A:(x, u) \mapsto \partial f(x) \times N_{\mathbb{R}_{+}^{p}} u$ is maximally monotone, $B_{1}:(x, u) \mapsto(\nabla h(x), 0)$ is $\beta$-cocoercive, and

$$
B_{2}:(x, u) \mapsto\left(\sum_{i=1}^{p} u_{i} \nabla g_{i}(x),-g_{1}(x), \ldots,-g_{p}(x)\right)
$$

is nonlinear, monotone and continuous [34]. If $Y \subset \operatorname{dom} \partial f \subset \cap_{i=1}^{p}$ int dom $g_{i}$ we have that $X:=Y \times \mathbb{R}_{+}^{p} \subset \operatorname{dom} A=\operatorname{dom} \partial f \times \mathbb{R}_{+}^{p} \subset \operatorname{dom} B_{2}=\cap_{i=1}^{p}$ int dom $g_{i} \times \mathbb{R}^{p}$ and, from [3, Corollary 25.5], we have that $A+B_{2}$ is maximally monotone. The method proposed in Theorem 2.3 reduces to

$$
(\forall k \in \mathbb{N}) \quad\left[\begin{array}{l}
y^{k}=\operatorname{prox}_{\gamma_{k} f}\left(x^{k}-\gamma_{k}\left(\nabla h\left(x^{k}\right)+\sum_{i=1}^{p} u_{i}^{k} \nabla g_{i}\left(x^{k}\right)\right)\right) \\
\text { For every } i=1, \ldots, p
\end{array} \mid \begin{array}{l}
\eta_{i}^{k}=\max \left\{0, u_{i}^{k}+\gamma_{k} g_{i}\left(x^{k}\right)\right\} \\
u_{i}^{k+1}=\max \left\{0, \eta_{i}^{k}-\gamma_{k}\left(g_{i}\left(x^{k}\right)-g_{i}\left(y^{k}\right)\right)\right\} \\
x^{k+1}=P_{Y}\left(y^{k}+\gamma_{k} \sum_{i=1}^{p}\left(u_{i}^{k} \nabla g_{i}\left(x^{k}\right)-\eta_{i}^{k} \nabla g_{i}\left(y^{k}\right)\right)\right),
\end{array}\right.
$$

where, for every $k \in \mathbb{N}, \gamma_{k}$ is found by the backtracking procedure defined in (2.9). Note that, since $B_{2}$ is nonlinear, the approaches proposed in [21, 42] cannot be applied to this instance.

In the particular instance when $f=\iota_{\Omega}$ for some nonempty closed convex set $\Omega$, we can choose, among other options, $Y=\Omega$ since we know that any solution must belong to $\Omega$. Moroever, when, for every $i \in\{1, \ldots, p\}, g_{i}: x \mapsto d_{i}^{\top} x$, where $d_{i} \in \mathbb{R}^{N}$, we have $B_{2}:(x, u) \mapsto\left(D^{\top} u,-D x\right)$, where $D=\left[d_{1}, \ldots, d_{p}\right]^{\top}$. This is a particular instance of problem (1.2) and $B_{2}$ is $\|D\|$-Lipschitz in this case, which allows us to use constant stepsizes $\left.\gamma_{k}=\gamma \in\right] 0, \chi[$, where $\chi$ is defined in (2.3) and $L=\|D\|$. Theorem 2.3 guarantees the convergence of the iterates $\left\{x^{k}\right\}_{k \in \mathbb{N}}$ thus generated to a solution to (6.19) in any case.

In the next section, we explore some numerical results showing the good performance of this method and the method with constant step-size when $g_{i}$ are affine linear.

7. Numerical simulations. In this section we provide two instances of Section 6.4 and we compare our proposed method with available algorithms in the literature.

7.1. Optimization with linear inequalities. In the context of problem (6.19), suppose that $\mathcal{H}=\mathbb{R}^{N}, h: x \mapsto\|A x-b\|^{2} / 2, A$ is a $m \times N$ real matrix with $N=2 m$ and $b \in \mathbb{R}^{m}, f=\iota_{[0,1]^{N}}$, and

$$
(\forall i \in\{1, \ldots, p\}) \quad g_{i}(x)=d_{i}^{\top} x,
$$

where $d_{1}, \ldots, d_{p} \in \mathbb{R}^{N}$. In this case, $B_{1}:(x, u) \mapsto\left(A^{\top}(A x-b), 0\right), B_{2}:(x, u) \mapsto$ $\left(D^{\top} u,-D x\right)$, where $D=\left[d_{1}, \ldots, d_{p}\right]^{\top}, \beta=\|A\|^{-2}$ and $L=\|D\|$. We compare the method proposed in (6.22) using the line search (FBHF-LS), the version with constant 


\begin{tabular}{|l||l|l|l||l|l|l||l|l|l|l||l||l||}
\hline \multicolumn{1}{|l||}{$\epsilon=10^{-7}$} & \multicolumn{3}{|c||}{ Tseng } & \multicolumn{3}{c||}{ FBHF } & \multicolumn{4}{c||}{ CV } & Tseng-LS & FBHF-LS \\
\hline$\delta, \bar{\sigma}$ & 0.8 & 0.9 & 0.99 & 3.2 & 3.99 & 4.4 & 0.0125 & 0.0031 & 0.0008 & 0.0002 & LS & LS \\
\hline$h\left(x^{*}\right)$ & 158.685 & 158.684 & 158.684 & 158.681 & 158.680 & 158.679 & 158.674 & 158.674 & 158.676 & 158.687 & 158.683 & 158.680 \\
\hline iter. & 20564 & 18482 & 16791 & 11006 & 8915 & 8243 & 9384 & 9158 & 8516 & 13375 & 14442 & 10068 \\
\hline time $(\mathrm{s})$ & 41.55 & 37.18 & 33.76 & 13.11 & 10.48 & 9.76 & 10.70 & 10.61 & 9.67 & 15.27 & 94.86 & 12.40 \\
\hline
\end{tabular}

TABLE 1

Comparison of Tseng, FBHF and CV (with different values of $\delta$ and $\bar{\sigma}$ ), Tseng-LS and FBHF$L S$ for a stop criterion of $\epsilon=10^{-7}$.

\begin{tabular}{|l|l|l|}
\hline$\epsilon=10^{-7}$ & av. iter. & av. time $(\mathrm{s})$ \\
\hline FBHF-LS & 36225 & 43.96 \\
\hline FBHF $(\delta=3.999)$ & 32563 & 39.07 \\
\hline FBHF $(\delta=4.7)$ & 28364 & 34.14 \\
\hline CV $(\sigma=0.0008)$ & 33308 & 38.60 \\
\hline
\end{tabular}

Average performance of the more efficient methods for 20 random realizations of $A, D$ and $b$ with $N=2000$ and $p=100$.

stepsize (FBHF), the method proposed by Condat and Vũ $[21,42]$ (CV), the method proposed by Tseng [40] with line search (Tseng-LS) and with constant stepsize (Tseng) for randomly generated matrices and vectors $A, D$ and $b$. We choose the same starting point for each method and the parameters for the line search for Tseng-LS and FBHFLS are $\theta=0.316, \varepsilon=0.88$ and $\sigma=0.9$. For the constant stepsizes versions of Tseng and FBHF, we use $\gamma=\delta /\left(\beta^{-1}+L\right)$ and $\gamma=\delta \beta /\left(1+\sqrt{1+16 \beta^{2} L^{2}}\right)$, respectively, and for $\bar{\sigma}>0$ we select $\tau=1 /\left(1 / 2 \beta+\bar{\sigma} L^{2}\right)$ in order to satisfy the convergence conditions on the parameters of each algorithm. We choose several values of $\delta$ and $\bar{\sigma}$ for studying the behavior and we use the stopping criterion $\left\|\left(x_{k+1}-x_{k}, u_{k+1}-u_{k}\right)\right\| /\left\|\left(x_{k}, u_{k}\right)\right\|<10^{-7}$. In Table 1 we show the performance of the five algorithms for random matrices $A$ and $D$ and a random vector $b$ with $N=2000$ and $p=100$ and a selection of the parameters $\sigma, \delta$. We see that for Tseng and FBHF the performance improve for larger choices of $\delta$, while for $\mathrm{CV}$ it is not clear how to choose $\bar{\sigma}$ in general. Even if the theoretical bound of FBHF does not permit $\delta$ to go beyond 4 , for $\delta=4.4$ the convergence is also obtained for this case with a better performance. We suspect that the particular structure of this particular case can be exploited for obtaining a better bound. We also observe that the best performance in time is obtained for the lowest number of iterations for each method. In addition, for this instance, algorithms FBHF, CV and FBHF-LS are comparable in computational time, while the algorithms by Tseng [40] are considerably less efficient in time and in number of iterations. In Table 2 we compare the average time and iterations that the more efficient methods in the first simulation take to achieve the stop criterion $\left(\epsilon=10^{-7}\right)$ for 20 random realizations of matrices $A$ and $D$ and a random vector $b$, with $N=2000$ and $p=100$. We use the parameters yielding the best performance of each method in the first simulation. For FBHF we also explore the case when $\delta=4.7$, which gives the best performance. We also observe that FBHF for $\delta=3.999$ is comparable with $\mathrm{CV}$ in average time, while FBHF-LS is slower for this instance.

7.2. Entropy constrained optimization. In the context of problem (6.19), suppose that $\mathcal{H}=\mathbb{R}^{N}, h: x \mapsto x^{\top} Q x-d^{\top} x+c, Q$ is a $N \times N$ semidefinite positive 
real matrix, $b \in \mathbb{R}^{N}, c \in \mathbb{R}, f=\iota_{\Omega}, \Omega$ is a closed convex subset of $\mathbb{R}^{N}, p=1$, and

$$
g_{1}: \mathbb{R}_{+}^{N} \rightarrow \mathbb{R}: x \mapsto \sum_{i=1}^{N} x_{i}\left(\ln \left(\frac{x_{i}}{a_{i}}\right)-1\right)-r,
$$

where $-\sum_{i=1}^{N} a_{i}<r<0, a \in \mathbb{R}_{++}^{N}$ and we use the convention $0 \ln (0)=0$. This problem appears in robust least squares estimation when a relative entropy constraint is included [31]. This constraint can be seen as a distance constraint with respect to the vector $a$, where the distance is measured by the Kullback-Leibler divergence [2].

In our numerical experience, we assume $Q=A^{\top} A, d=A^{\top} b$ and $c=\|b\|^{2} / 2$, where $A$ is a $m \times N$ real matrix with $N=2 m$ and $b \in \mathbb{R}^{m}$, which yields $h: x \mapsto$ $\|A x-b\|^{2} / 2, \beta=\|A\|^{-2}, \Omega=[0.001,1]^{N}$, and $a=(1, \ldots, 1)^{\top}$. In this context, $g_{1}$ achieves its minimum in $\bar{x}=(1, \ldots, 1)^{\top}$ and $g_{1}(\bar{x})=-N$ and we choose $r \in$ ] - $N, 0[$. Since the constraint is not linear, we cannot use the methods proposed in $[42,21]$. We compare the method proposed in (6.22) with line search (FBHF-LS) with the Tseng's method with linesearch [40] (Tseng-LS) and two routines in matlab: fmincon.interior-point (FIP) and fmincon.sqp (SQP). For $m=100,200,300$, we generate 20 random matrices $A$ and random vectors $b$ and we compare the previous methods by changing $r \in\{-0.2 N,-0.4 N,-0.6 N,-0.8 N\}$ in order to vary the feasible regions. We choose the same starting point for each method and the parameters for the line search for Tseng-LS and FBHF-LS are $\theta=0.707, \varepsilon=0.88$ and $\sigma=0.9$. The stopping criterion is $\left\|\left(x_{k+1}-x_{k}, u_{k+1}-u_{k}\right)\right\| /\left\|\left(x_{k}, u_{k}\right)\right\|<\epsilon$ with $\epsilon=10^{-11}$. In Table 3 we show, for $m=300$, the value of the objective function $h$, the nonlinear constraint $g_{1}$ and time for achieving the stopping criterion for a fixed random matrix $A$ and vector $b$ by moving $r \in\{-0.2 N,-0.4 N,-0.6 N,-0.8 N\}$. We observe that all methods achieve almost the same value of the objective function and satisfy the constraints, but in time FBHF-LS obtains the best performance, even if the number of iterations are larger than that of FIP and SQP. Tseng-LS has also a better performance in time than FIP and SQP, with a much larger number of iterations. We also observe that, the smaller the feasible set is, the harder is for all the methods to approximate the solution and the only case when the constraint is inactive is when $r=-0.2 N$. On the other hand, even if in the cases $r=-0.6 N$ and $r=-0.8 N$ we have $g_{1}\left(x^{*}\right)>0$, the value is $\approx 10^{-6}$ which is very near to feasibility. This behavior is confirmed in Table 4 , in which we show, for each $m \in\{100,200,300\}$, the average time and iterations obtained from the 20 random realizations by moving $r \in\{-0.2 N,-0.4 N,-0.6 N,-0.8 N\}$. We observe that FBHF-LS takes considerably less time than the other algorithms to reach the stopping criterion and the difference is more when dimension is higher. Since FIP and SQP are very slow for high dimensions, in Table 5 we compare the efficiency of Tseng-LS and FBHF-LS for 20 random realizations of $A$ and $b$ with $N \in\{1000,2000,3000\}$ for $r=-0.4 N$ and $\epsilon=10^{-5}$. The computational time of both methods are reasonable, but again FBHF-LS is faster. FBHF-LS use less iterations than Tseng-LS for achieving the same criterion and, even if we reduce $\epsilon$ from $10^{-5}$ to $10^{-10}$ and the number of iterations are more than 3 times that of Tseng-LS for the weaker criterion, the computational time is similar. We also observe that the percentage of relative improvement of an algorithm $A$ with respect to TsengLS, measured via \%imp. $(A)=100 *\left(f\left(x_{A}\right)-f\left(x_{T}\right)\right) / f\left(x_{T}\right)$, where $x_{T}$ and $x_{A}$ are the approximative solutions obtained by Tseng-LS and $A$, is bigger for smaller dimensions. For instance, in the case $500 \times 1000$, FBHF-LS obtain an approximative solution for which the objective function has a $12 \%$ of relative improvement with respect to that of 


\begin{tabular}{|c|c|c|c|c|c|c|c|c|c|c|c|c|c|c|c|c|}
\hline & \multicolumn{4}{|c|}{$r=-0.2 N$} & \multicolumn{4}{|c|}{$r=-0.4 N$} & \multicolumn{4}{|c|}{$r=-0.6 N$} & \multicolumn{4}{|c|}{$r=-$} \\
\hline $300 \times 600$ & $h\left(x^{*}\right)$ & $g_{1}\left(x^{*}\right)$ & time $(\mathrm{s})$ & \begin{tabular}{|l|} 
iter. \\
\end{tabular} & $h\left(x^{*}\right)$ & $g_{1}\left(x^{*}\right)$ & time (s) & \begin{tabular}{|l|l|} 
iter. \\
\end{tabular} & $h\left(x^{*}\right)$ & $g_{1}\left(x^{*}\right)$ & time $(\mathrm{s})$ & iter. & $h\left(x^{*}\right)$ & \begin{tabular}{|l}
$g_{1}\left(x^{*}\right)$ \\
\end{tabular} & time $(\mathrm{s})$ & iter. \\
\hline & & & & 562 & & & & 574 & & & & & & & & \\
\hline & & & & 357 & & & & & & & & & & & & \\
\hline & & & & 137 & & & & 30110 & & & 44.248 & & & & & 75254 \\
\hline & $2.15 \mathrm{E}-15$ & -119.338 & 1.053 & & $5.56 \mathrm{E}-15$ & $-4.71 \mathrm{E}-09$ & 2.220 & 21106 & 244.551 & $1.10 \mathrm{E}-06$ & 10.381 & 19492 & 4075.6822 & $2.07 \mathrm{E}-06$ & 17.464 & 60442 \\
\hline
\end{tabular}

TABLE 3

Comparison of objective function and constraints values, time and number of iterations of FIP, $S Q P$, Tseng-LS and FBHF-LS algorithms for solving the entropy constrained optimization when $N=600, m=300$ and $r \in\{-0.2 N,-0.4 N,-0.6 N,-0.8 N\}$.

\begin{tabular}{|c|c|c|c|c|c|c|c|c|c|c|c|c|}
\hline Time (s) & \multicolumn{4}{|c|}{$100 \times 200$} & \multicolumn{4}{|c|}{$200 \times 400$} & \multicolumn{4}{|c|}{$300 \times 600$} \\
\hline constraint & $r=-0.2 N$ & $r=-0.4 N$ & $r=-0.6 N$ & $=-0.8 \mathrm{~N}$ & $r=-0.2 N$ & $r=-0.4 N$ & $r=-0.6 \mathrm{~N}$ & $r=-0.8 N$ & $r=-0.2 N$ & $r=-0.4 N$ & $r=-0.6 N$ & $r=-0.8 N$ \\
\hline & 8.24 & 9.50 & 11.92 & 11.22 & 52.95 & 57.92 & 75.02 & 76.29 & 142.51 & 183.22 & 253.80 & 324.42 \\
\hline & 8.60 & 11.18 & 14.28 & 18.51 & & 98.70 & 122 & 209 & & 489 & 569.34 & 1075.42 \\
\hline Tseng-LS & 22.92 & 10.71 & 7.92 & 9.91 & 81.16 & 13.46 & 39.17 & 83.01 & 139.47 & 26.50 & 84.07 & 111.44 \\
\hline FBHF-LS & 2.21 & 0.99 & 2.72 & 2.51 & 7.06 & 0.95 & 10.23 & 18.09 & 12.48 & 1.88 & 20.59 & 18.30 \\
\hline
\end{tabular}

TABLE 4

Average time (s) to reach the stopping criterion of 20 random realizations for FIP, SQP, Tseng-LS and FBHF-LS for a matrix A with dimension $100 \times 200,200 \times 400$ and $300 \times 600$ and $r \in\{-0.2 N,-0.4 N,-0.6 N,-0.8 N\}$.

Tseng-LS for $\epsilon=10^{-5}$ and, if the criterion is strengthened to $10^{-10}$, the improvement raises to $20 \%$. For higher dimensions, this quantities are considerably reduced.

8. Conclusion. In this paper, we systematically investigated a new extension of Tseng's forward-backward-forward method and the forward-backward method. The three primary contributions of this investigation are (1) a lower per-iteration complexity variant of Tseng's method which activates the cocoercive operator only once; (2) the ability to incorporate variable metrics in operator-splitting schemes, which, unlike typical variable metric methods, do not enforce compatibility conditions between metrics employed at successive time steps; and (3) the ability to incorporate modified resolvents $J_{P^{-1} A}$ in iterative fixed-point algorithms, which, unlike typical preconditioned fixed point iterations, can be formed from non self-adjoint linear operators $P$, which lead to new Gauss-Seidel style operator-splitting schemes.

Acknowledgments: This work is partially supported by NSF GRFP grant DGE-0707424, by CONICYT grant FONDECYT 11140360, and by "Programa de financiamiento basal" from CMM, Universidad de Chile. We want to thank the two anonymous reviewers, whose comments and concerns allowed us to improve the quality of this manuscript.

\section{REFERENCES}

[1] J.-B. BAillon AND G. HAdDAD, Quelques propriétés des opérateurs angle-bornés et ncycliquement monotones, Israel Journal of Mathematics, 26 (1977), pp. 137-150.

[2] M. Basseville, Divergence measures for statistical data processing-an annotated bibliography, Signal Processing, 93 (2013), pp. $621-633$.

[3] H. H. Bauschke and P. L. Combettes, Convex analysis and monotone operator theory in Hilbert spaces, Second Edition, Springer, 2017.

[4] J. Y. Bello Cruz and T. T. A. NGhia, On the convergence of the forward-backward splitting method with linesearches, Optim. Methods Softw., 31 (2016), pp. 1209-1238.

[5] D. P. Bertsekas, Incremental proximal methods for large scale convex optimization, Mathematical Programming, 129 (2011), pp. 163-195.

[6] - Incremental Aggregated Proximal and Augmented Lagrangian Algorithms, arXiv preprint arXiv:1509.09257, (2015).

[7] P. BIANCHI, A stochastic proximal point algorithm: convergence and application to convex 


\begin{tabular}{|c|l||l|l|l||l|l|l||l|l|l|}
\hline & \multicolumn{1}{|c||}{} & \multicolumn{3}{c||}{$500 \times 1000$} & \multicolumn{3}{c||}{$1000 \times 2000$} & \multicolumn{3}{c|}{$1500 \times 3000$} \\
\hline stop crit. & Algorithm & time (s) & iter. & $\%$ imp. & time (s) & iter. & $\%$ imp. & time (s) & iter. & $\%$ imp. \\
\hline \multirow{2}{*}{$10^{-5}$} & Tseng-LS & 17.22 & 2704 & 0 & 52.80 & 4027 & 0 & 91.23 & 3349 & 0 \\
& FBHF-LS & 2.33 & 1993 & 12.2 & 6.26 & 3239 & 3.2 & 9.56 & 2474 & 0.1 \\
\hline $10^{-10}$ & FBHF-LS & 10.67 & 10092 & 20.1 & 71.51 & 33637 & 6.5 & 53.47 & 12481 & 0.2 \\
\hline
\end{tabular}

TABLE 5

Comparison between Tseng-LS and FBHF-LS for higher dimensions. We compare average time and average number of iterations for achieving stop criteria together with percentage of relative improvement with respect to Tseng-LS approximate solution.

optimization, in IEEE 6th International Workshop on Computational Advances in MultiSensor Adaptive Processing, 2015, pp. 1-4.

8] L. M. Briceño-Arias and P. L. Combettes, A monotone+ skew splitting model for composite monotone inclusions in duality, SIAM Journal on Optimization, 21 (2011), pp. 1230-1250.

[9] L. M. Briceño-Arias, D. Kalise, and F. J. Silva, Proximal methods for stationary mean field games with local couplings, https://arxiv.org/abs/1608.07701, (2017).

[10] R. E. BRUCK, An iterative solution of a variational inequality for certain monotone operators in hilbert spaces, Bull. Amer. Math. Soc., 81 (1975), pp. 890-892.

[11] L. CAfarelLi, The obstacle problem revisited, The Journal of Fourier Analysis and Applications, 4 (1988), pp. 383-402.

[12] L. A. Caffarelli, The obstacle problem, Lezioni Fermiane. Accademia Nazionale dei Lincei, Rome; Scuola Normale Superiore, Pisa, 1998.

[13] A. Chambolle And T. Pock, A first-order primal-dual algorithm for convex problems with applications to imaging, Journal of Mathematical Imaging and Vision, 40 (2011), pp. 120145.

[14] F. R. Chung, Spectral graph theory, no. 92, American Mathematical Soc., 1997.

[15] P. L. Combettes, Quasi-Fejérian analysis of some optimization algorithms, Studies in Computational Mathematics, 8 (2001), pp. 115-152.

[16] - Solving monotone inclusions via compositions of nonexpansive averaged operators, Optimization, 53 (2004), pp. 475-504.

[17] P. L. Combettes And J.-C. Pesquet, Primal-dual splitting algorithm for solving inclusions with mixtures of composite, lipschitzian, and parallel-sum type monotone operators, SetValued and variational analysis, 20 (2012), pp. 307-330.

[18] P. L. Combettes And B. C. VŨ, Variable metric quasi-fejér monotonicity, Nonlinear Analysis: Theory, Methods \& Applications, 78 (2013), pp. 17-31.

[19] P. L. Combettes And B. C. VŨ, Variable metric forward-backward splitting with applications to monotone inclusions in duality, Optimization, 63 (2014), pp. 1289-1318.

[20] P. L. Combettes And V. R. Wajs, Signal recovery by proximal forward-backward splitting, Multiscale Model. Simul., 4 (2005), pp. 1168-1200.

[21] L. Condat, A primal-dual splitting method for convex optimization involving Lipschitzian, proximable and linear composite terms, Journal of Optimization Theory and Applications, 158 (2013), pp. 460-479.

[22] D. DAvIS, Convergence rate analysis of primal-dual splitting schemes, arXiv preprint arXiv:1408.4419, (2014).

[23] A. Defazio, F. Bach, and S. Lacoste-Julien, SAGA: A Fast Incremental Gradient Method With Support for Non-Strongly Convex Composite Objectives, in Advances in Neural Information Processing Systems, 2014, pp. 1646-1654.

[24] A. Defazio, J. Domke, and T. Caetano, Finito: A Faster, Permutable Incremental Gradient Method for Big Data Problems, in Proceedings of the 31st International Conference on Machine Learning (ICML-14), 2014, pp. 1125-1133.

[25] A. Goldstein, Convex programming in hilbert spaces, Bull. Amer. Math. Soc., 70 (1964), pp. 709-710.

[26] P. Grisvard, Elliptic problems in nonsmooth domains, Pitman, Boston, MA, 1985.

[27] B. HE AND X. YUAN, Convergence analysis of primal-dual algorithms for a saddle-point problem: From contraction perspective, SIAM Journal on Imaging Sciences, 5 (2012), pp. 119149.

[28] Y. Huang And Y. Dong, New properties of forward-backward splitting and a practical proximal-descent algorithm, Appl. Math. Comput., 237 (2014), pp. 60-68.

[29] R. Johnson And T. Zhang, Accelerating Stochastic Gradient Descent Using Predictive Vari- 
ance Reduction, in Advances in Neural Information Processing Systems, 2013, pp. 315-323.

[30] P. LATAFAT AND P. Patrinos, Asymmetric forward-backward-adjoint splitting for solving monotone inclusions involving three operators, https://arxiv.org/abs/1602.08729, (2016).

[31] B. C. Levy And R. Nikoukhah, Robust least-squares estimation with a relative entropy constraint, IEEE Transactions on Information Theory, 50 (2004), pp. 89-104.

[32] P.-L. Lions AND B. Mercier, Splitting algorithms for the sum of two nonlinear operators, SIAM Journal on Numerical Analysis, 16 (1979), pp. 964-979.

[33] A. Nedic and D. P. Bertsekas, Incremental subgradient methods for nondifferentiable optimization, SIAM Journal on Optimization, 12 (2001), pp. 109-138.

[34] R. T. RockAFELlar, Monotone operators associated with saddle-functions and minimax problems, in Nonlinear Functional Analysis, Part 1 F. E. Browder (Ed.), Proc. Sympos. Pure Math., vol. 18, AMS, Providence, RI, 1970, pp. 241-250.

[35] S. SALzO, The variable metric forward-backward splitting algorithm under mild differentiability assumptions, SIAM Journal on Optimization, 27 (2017), pp. 2153-2181.

[36] M. Schmidt, N. L. Roux, And F. BACH, Minimizing finite sums with the stochastic average gradient, arXiv preprint arXiv:1309.2388, (2013).

[37] S. Shalev-Shwartz and S. Ben-David, Understanding Machine Learning: From Theory to Algorithms, Cambridge University Press, New York, NY, USA, 2014.

[38] W. Shi, Q. Ling, G. Wu, And W. Yin, Extra: An exact first-order algorithm for decentralized consensus optimization, SIAM Journal on Optimization, 25 (2015), pp. 944-966.

[39] — A proximal gradient algorithm for decentralized composite optimization, IEEE Transactions on Signal Processing, 63 (2015), pp. 6013-6023.

[40] P. TSEnG, A modified forward-backward splitting method for maximal monotone mappings, SIAM Journal on Control and Optimization, 38 (2000), pp. 431-446.

[41] B. C. VŨ, A variable metric extension of the forward-backward-forward algorithm for monotone operators, Numer. Funct. Anal. Optim., 34 (2013), pp. 1050-1065.

[42] B. C. VŨ, A splitting algorithm for dual monotone inclusions involving cocoercive operators, Advances in Computational Mathematics, 38 (2013), pp. 667-681.

[43] M. Wang and D. P. Bertsekas, Stochastic First-Order Methods with Random Constraint Projection, SIAM Journal on Optimization, 26 (2016), pp. 681-717.

[44] E. ZeIDLER, Nonlinear Functional Analysis and its Applications II/B - Nonlinear Monotone Operators, Springer, New York, 1990.

[45] C. ZăLinescu, Convex analysis in general vector spaces, World Scientific Publishing Co., Inc., River Edge, NJ, 2002 